\title{
Informalität von oben und unten
}

Stadtentwicklung in der afghanischen Hauptstadt Kabul nach der westlichen Intervention ist gekennzeichnet von der Verschränkung einer durch Staatsaufbau nach westlichem Vorbild gesteuerten Logik und der sich in diesem Rahmen ausbreitenden Aneignung des städtischen Raumes durch ganz verschiedene Akteure. Vor diesem Hintergrund wird das Ziel verfolgt, stadträumliche Entwicklungsprozesse in Kabul nach 2001 genauer in den Blick zu nehmen und mit jüngeren Debatten um urbane Informalität im globalen Süden zu verknüpfen. Dabei werden verschiedene, aufeinander bezogene Regime der Stadtplanung und ihre Raumproduktionen unter der Linse urbaner Informalität genauer betrachtet. Es soll herausgearbeitet werden, wie eine Herstellung und Zementierung städtischer Ungleichheit forciert wird, wie bestimmte soziale Dispositionen und Praktiken im Kontext von Informalität charakterisiert werden und wie urbane Informalität gezielt als Ressource zur Macht- und Wohlstandsaneignung eingesetzt werden kann.

Die Stadtentwicklung in der afghanischen Hauptstadt Kabul nach der westlichen Invasion im Herbst des Jahres 2001 ist gekennzeichnet von der Verschränkung einer durch einen Staatsaufbau nach westlichem Vorbild gesteuerten Logik und der sich in diesem Rahmen ausbreitenden Aneignung des städtischen Raumes, sowohl durch internationale und afghanische Eliten als auch durch eine von oftmals extremer Armut und hoher Verwundbarkeit betroffene Bevölkerung. Die resultierende, Überdetermination' der städtischen Entwicklung in Kabul (Esser 2013) wird konturiert durch Landspekulation und Landnahme, einen modernisierenden und wirtschaftsliberalen Entwicklungsnarrativ, offizielle stadtplanerische Vorgaben und deregulierten Siedlungsbau. Dazu kommt ein enormes und vornehmlich durch Rückkehr von Geflüchteten und Land-Stadt-Migration getragenes Bevölkerungswachstum. Diese verschiedenen und sich wechselseitig beeinflussenden und oftmals widersprechenden Faktoren der politischen und ökonomischen Stadtentwicklung Kabuls spiegeln sich in der Ambiguität der Afghanistanintervention selber (Kühn 2014) und resultieren in neuen lokalen Konfliktlagen, einer Machtkonsolidierung ehemaliger Kriegsfürsten und, damit einhergehend, der politischen und wirtschaftlichen Marginalisierung einer städtischen Mehrheit in Kabul. 
Vor diesem komplexen Hintergrund militärisch-humanitärer Intervention (Suhrke 2011) und interner machtpolitischer Gemengelagen (Giustozzi 2009, Mukhopadhyay 2014) zur Beförderung einer elitären Aneignung der Stadt wird in diesem Beitrag das Ziel verfolgt, stadträumliche Entwicklungsprozesse in Kabul nach 2001 genauer in den Blick zu nehmen und mit jüngeren Debatten um urbane Informalität im globalen Süden zu verknüpfen. Dabei geht es vornehmlich darum, verschiedene, sich teilweise widersprechende, aber gleichzeitig aufeinander bezogenen, Regime der Stadtplanung und ihrer Raumproduktionen unter der Linse urbaner Informalität genauer zu betrachten. In ihrem Zusammenspiel führt die gesellschaftliche Praxis dieser Planungsregime selber bereits zu einer überdeterminierten Situation, in der machtvolle Interessen städtischer Raumproduktion abgebildet und konfliktgeladene Spannungsfelder sichtbar werden.

\section{Informalität als ,Idiom der Urbanisierung' und städtische Planungsregime in Kabul}

Die Überlegungen von Ananya Roy (2005, 2009, 2011) zu urbaner Informalität bieten einen relevanten analytischen Zugang, um die Situation in Kabul besser nachzuvollziehen. Informalität als raumproduzierende Praxis ist demnach kein bloßes Phänomen von Armut und Marginalisierung (wie z. B. im Sinne von Davis 2006) und formt auch nicht ausschließlich den „Habitus der Enteigneten“ (Bayat 2007) oder eine zentrale global-städtische Lebensweise (AlSayyad 2004). Diese Konzeptualisierungen stellen äußerst wichtige Überlegungen zur Beschreibung und Analyse der prekären Situation einer globalen städtischen Mehrheit dar, doch Informalität ist ebenso eine machtvolle Strategie zum Beispiel zur unsanktionierten Aneignung von Land oder des profitorientieren Wohnungsbaus. Der Unterschied besteht eben in der Duldung und Förderung elitärer Informalität, von oben und der Illegalisierung oder Kriminalisierung subalterner Praktiken der Raumaneignung. Die begrifflichen Grenzen verschwimmen, und Informalität ist nicht das Gegenstück der formalisierten Stadt, sondern sowohl eine ungleiche Austauschbeziehung, die verschiedene ökonomische Tätigkeiten und städtische Räume miteinander verbindet, als auch ein Ausdruck städtischer Herrschaft, verankert im staatlichen Planungs- und Gesetzesapparat (Roy 2005: 148f.). In dieser Weise kann Informalität als Modus der Urbanisierung verstanden werden (ebd.). Konzeptionell wird der Begriff zum ,...heuristischen Hilfsmittel der Dekonstruktion staatlicher Legitimität und seiner Instrumente: Karten, Vermessungen, Eigentum, Zonierungen und - ganz wichtig - das Gesetz“ (Roy 2011: 233; Übers. d. A.). Damit lässt sich nachvollziehen, wie sich machtgesteuerte Interpretationen von Planungskarten, von Eigentumsverhältnissen und von gesetzlichen Grundlagen flexibel einsetzen lassen, um bestimmte Interessen durchsetzen zu können und um Legitimität, Legalität und städtische Regulierung zu verhandelbaren Entitäten werden zu lassen - staatliche Stadtpolitik selbst wird informal (Roy 2009). Die damit deutlich werdende fehlende Erklärungskraft einer im politischen Diskurs nach wie vor üblichen Dichotomisierung von formalen und informalen städtischen Verhältnissen mit Blick auf eine angemessenere Konzeptualisierung „hybrider formal-informaler Arrangements“ 
(Altrock 2012) lässt sich am empirischen Beispiel von Kabul sehr gut nachvollziehen. Dabei bieten auch die von McFarlane und Waibel (2012) aufgestellten Betrachtungsebenen des „formal-informalen Spannungsfeldes“ wichtige Aspekte. Die Charakterisierung dieses Spannungsfeldes von (In-) Formalität als räumliche Kategorisierung, als organisatorische Form, als staatliches Werkzeug und als verhandelbares Gut ist für eine übergeordnete Einschätzung der Planungsrealität in Kabul übertragbar.

Mit diesen Bezügen zu einem angemesseneren Verständnis urbaner Formalität und Informalität als komplexe stadtpolitische Praktiken soll herausgearbeitet werden, wie in Kabul eine Herstellung und Zementierung städtischer Ungleichheit forciert wird, wie bestimmte soziale Dispositionen und Praktiken im Kontext von Informalität charakterisiert werden, und wie urbane Informalität gezielt als Ressource zur Macht- und Wohlstandsaneignung eingesetzt werden kann.

Im Kontext der Situation in Kabul lassen sich diese grundsätzlichen konzeptionellen Überlegungen auf eine analytische Unterscheidung zwischen drei verschiedenen und koexistenten Planungsregimen übertragen, die in ihrem Zusammenspiel die Stadtentwicklung seit 2001 steuern. Diese Formen von Stadtplanung werden hier schlagwortartig mit Blick auf die dominanten und symbolisierenden Baumaterialien in den unterschiedlichen Formen als ,Beton‘, ,Lehm‘ und ,Spiegelglas‘ bezeichnet.

,Beton' als die erste Perspektive behandelt die offizielle, formalisierte Politik in Kabul und bildet die Domäne der Technokraten und Beamten in den nationalen Behörden wie der Stadtverwaltung (Kabul Municipality) und dem Ministerium für Stadtentwicklung und Wohnungsbau, die in einer von Konkurrenz und Kooperation gekennzeichneten Gemengelage versuchen, eine formalisierte, von westlichen Beratern unterstützte und auf den bestehenden gesetzlichen Grundlagen beruhende Stadt- und Planungspolitik durchzuführen. Diese nahm ihren Anfang in den 1920er Jahren unter der modernisierenden Agenda des damaligen Königs Amanullah und basiert auf sukzessiven Masterplänen, die mit sowjetischer Unterstützung und versuchter Einflussnahme durch die USA in einem Wettstreit um Entwicklung seit den 1960er Jahren als wesentliches Planungsinstrument dienen (Beyer 2012). Die heutige formelle Planungsgrundlage städtischer Behörden ist der mittlerweile anachronistische, im Jahre 1978 mit sowjetischer Unterstützung fertiggestellte, Masterplan für eine Hauptstadt mit zwei Millionen Einwohnern. Auch wenn die Notwendigkeit einer Revision dieses Plans erkannt wurde, sieht die dadurch vermittelte Stadtpolitik die Realität der weitschweifenden informellen Siedlungen in Kabul nicht vor. Hier wird eine klare Dichotomisierung zwischen formellen und informellen räumlichen Kategorisierungen und Organisationsformen aufrechterhalten und damit städtische Modernität affirmiert und Illegalität definiert (McFarlane/Waibel 2012).

,Lehm` als die zweite Perspektive bezieht sich auf ,Informalität von unten' und bezeichnet eben diese durch offizielle Stadtpolitik illegalisierte und durch die internationale Entwicklungspolitik weitestgehend ignorierte Form von Planung. Die mit ,Lehm` verknüpften Prozesse haben dabei ein enormes städtisches Bevölkerungswachstum aufgefangen und Alltag und Überleben in Wohnvierteln ohne grundlegende Infrastruktur ermöglicht. Offizielle Daten beziffern das Bevölkerungswachstums mit jährlich 
11,6 Prozent zwischen den Jahren 2000 und 2015, sodass Kabul mit einer offiziell gezählten Einwohnerschaft von knapp 3,7 Millionen (Government of the Islamic Republic Afghanistan 2015) die am schnellsten wachsende Stadt in der gesamten Region Süd- und Zentralasiens ist. Dabei sind diese Zahlen vermutlich noch deutlich zu niedrig angesetzt - andere Angaben gehen von einer Bevölkerung von deutlich über fünf Millionen Menschen aus (Foschini 2017). Das städtische Wachstum ist im Wesentlichen getragen durch die Urbanisierung von Armut in dem vom Siedlungsprogramm der Vereinten Nationen so bezeichneten „urban penalty“ (UN Habitat 2006: 107). Dies bedeutet, dass der durch Rückkehr von Geflüchteten und Landflucht getragene Bevölkerungszuwachs dazu führt, dass die städtische Armut stark zunimmt und im Vergleich zu ländlichen Siedlungen die Gesundheitsvorsorge, der Bildungsstand, die Sterblichkeitsraten und Beschäftigungsmöglichkeiten vergleichsweise noch schlechter ausfallen.

,Lehm' bildet den Bereich der städtischen Mehrheit und stellt den Schauplatz eines alltäglichen Existenzkampfes unter oftmals extremen Lebensbedingungen, gekennzeichnet durch Flexibilität und Pragmatismus. Die hiermit verbundene informelle Planung nimmt ihren langsamen Anfang in den 1970er Jahren, als erste städtische Südhanglagen in Zentrumsnähe von erfolgreichen Arbeitsmigranten bebaut wurden (Bechhoefer 1977). Mit dem Abzug der Sowjets 1989 und dem Zusammenbruch der kommunistischen Regierung 1992 nach der Ermordung des Staatschefs Najibullah beginnt ein blutiger Bürgerkrieg und ein gewaltsamer Kampf um die Kontrolle von Kabul zwischen den verschiedenen und zunehmend ethnisierten MudschahedinFraktionen, der zu weitreichenden Zerstörungen von Bausubstanz und Infrastruktur führte (Dorronsoro 2000, 2007). Der Zerfall des Kabul-Regimes unter Najibullah markiert gleichzeitig den Beginn eines rapide zunehmenden informellen Wachstums der Stadt und eine enorme Ausdehnung neuer und nicht zentral geplanter Siedlungen. Eine lang andauernde extreme Dürreperiode (1998-2001) fast im gesamten Land (Kogan 2002) befeuerte das informelle Wachstum, ebenso wie die Rückkehr von Geflüchteten aus Pakistan und dem Iran nach der Vertreibung der Taliban (Turton/Marsden 2002).

,Spiegelglas' als die dritte Perspektive städtischer Planung betrachtet, wie machtvolle Akteure den Stadtraum Kabuls nach 2001 für sich in Anspruch nehmen und dabei im Sinne einer ,Informalität von oben ' offizielle Regelungen ungestraft ignorieren. Dies betrifft Landnahmen und Vertreibungen von Armutsgruppen durch Kriegsfürsten oder Parlamentsmitglieder, die neue Luxusviertel errichten lassen, aber auch die Politik von internationalen Geberorganisationen, Botschaften oder des Militärs, die ganze Stadtgebiete Kabuls zu einer von normalen Stadtbewohnern nicht mehr betretbaren Sicherheitszone werden lassen. Als exzeptionalistische Planung wird Dominanz in eine materielle Form überführt, die den Interessen lokaler Machthaber, aber auch der internationalen Entwicklungsindustrie dient. Hier lässt sich Informalität als „verhandelbares Gut“ (McFarlane/Waibel 2012) verstehen.

In der Zusammenschau bieten diese drei Perspektiven einen Blick auf dauerhafte Raumproduktionen in Kabul, welche die dominante Politik und den prekären Alltag einer städtischen Mehrheit miteinander verknüpfen. Die Pluralisierung von koexistenten Planungsregimen in Kabul ist in Abbildung 1 graphisch illustriert und in Verbindung gesetzt mit den einschneidenden 
Abb. 1 Pluralisierung von Stadtplanung in Kabul (Entwurf: Calogero 2011)

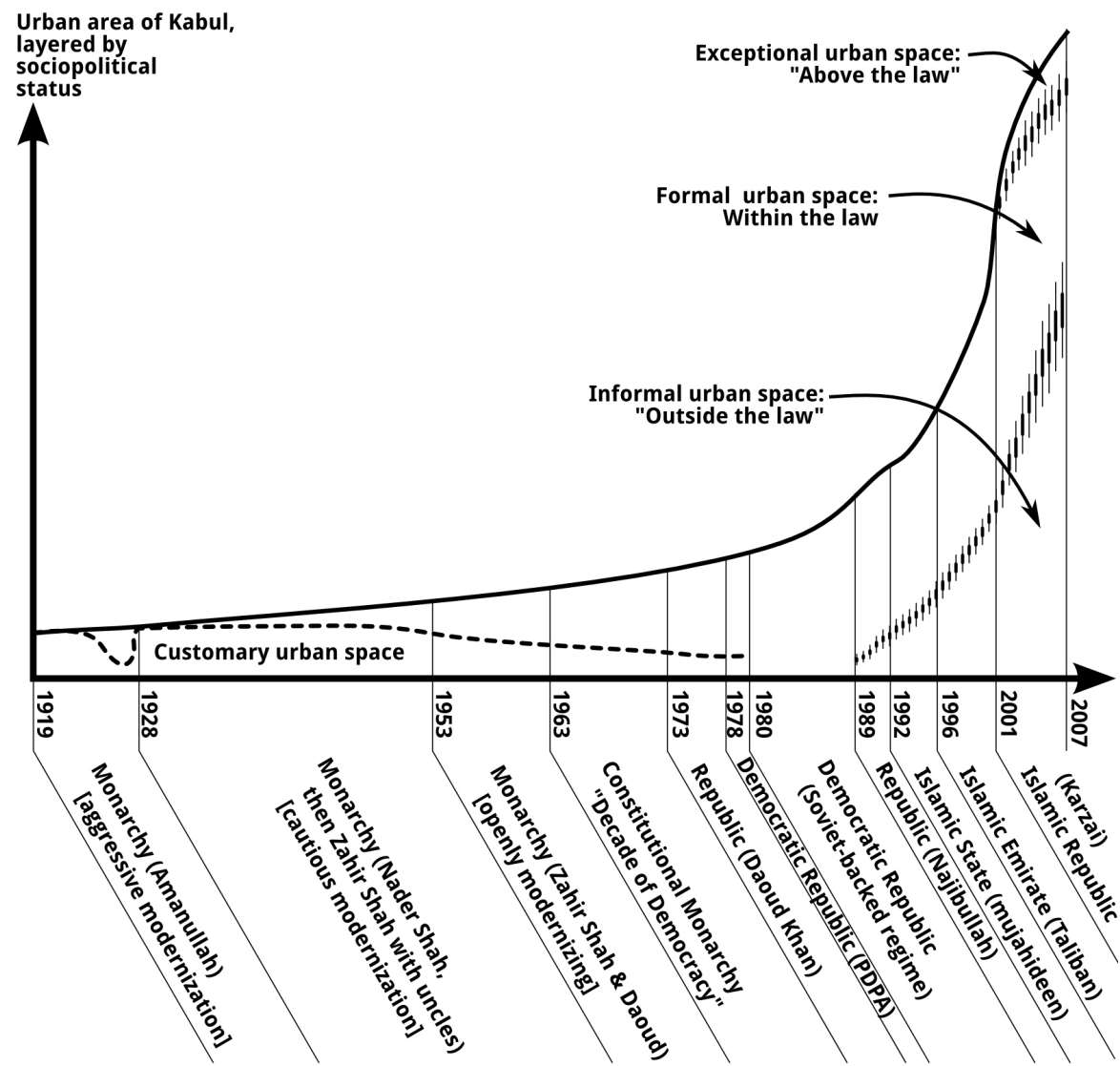

politischen Veränderungen in der Geschichte Afghanistans. Die Abbildung symbolisiert dabei die politische Bedeutung städtischen Raums und zeigt die ungefähren Proportionen der im Kontext eines jeweiligen Planungsregimes bebauten Fläche (Calogero 2011). Die lange Kontinuität formaler und durch Behörden gesteuerter Stadtplanung mit Blick auf eine moderne Stadtentwicklung Kabuls wird hier deutlich, ebenso wie der rapide und zunehmende Anstieg informeller Planung seit 1992 und die erst nach der westlichen Invasion im Jahre 2001 in erheblichen Maße einsetzende exzeptionalistische Planung als Informalität von oben. Letztere hat allerdings nach 2007 eine enorme und in der Grafik nicht repräsentierte Erweiterung in Form neu errichteter Stadtteile für wohlhabende Schichten erfahren (sogenannte shahraks).

Basis der folgenden Ausführungen zu den Modi jeweiliger Planungsformen und den daraus resultierenden Raumentwicklungen bilden eigene empirische Untersuchungen in Kabul zwischen 2004 und 2007 in verschiedenen informellen Siedlungen (Schütte 2006, Beall/Schütte 2006) und Interviews mit Vertretern der Stadtverwaltung und des Ministeriums für Stadtentwicklung in Kabul (Calogero 2011). Entsprechend bilden die Ausführungen die aktuelle Situation nicht umfassend ab, sondern sind auf den Zeitraum bis 2010 fokussiert. Ergänzende Beobachtungen und weitere Gespräche mit Vertretern verschiedener Behörden in 2017 versuchen allerdings jüngere Entwicklungen wie das Aufkommen von shahraks mit einzubeziehen.

\section{Beton als formelle Planung:}

\section{Affirmation von Modernität, Definition von Illegalität}

Die politisch-militärische und humanitäre Intervention in Afghanistan wird begleitet und unterstützt von einer diskursiven Besatzung, die drei Tropen 
bemüht: leidende afghanische Frauen, eine archaische und deterministische Kultur, und islamischer Fundamentalismus als die Wurzel allen Übels im Lande (Daulatzai 2008: 420). Im vorliegenden Kontext muss dieser Liste die westliche Wahrnehmung Afghanistans als ,anti-modernes“ Land hinzugefügt werden, dem nur mit vereinten internationalen Kräften zum Fortschritt verholfen werden kann. Diese Denkweise vergisst die Modernisierungsbemühungen des Entwicklungsstaates Afghanistan in Zeiten von Monarchie und Republik (Gregorian 1969, Poullada 1973, Schinasi 2017), der sowohl durch die Sowjetunion (Beyer 2012, Dörre/Kraudzun 2012) als von durch die USA (Cullather 2002) und Deutschland in seinen Fortschrittsinszenierungen unterstützt wurde (Kreutzmann/Schütte 2010, Ostermeier 2017). Die April-Revolution im Jahre 1978 und die anschlieBende sowjetische Besatzung von 1979 bis 1989 markierten das Ende dieses Entwicklungsmodells (Misdaq 2006).

Die praktische Implementierung von Stadtplanung im Zeichen des kalten Krieges gehorchte dem Zeitgeist der 1960er Jahre und setzte auf die Modernisierung, gesteuert durch klar formulierte Vorgaben im Rahmen von Masterplänen. Hier waren sowjetische Berater und die im Jahre 1964 gegründete „Central Authority for Housing and Town Planning“ in den entscheidenden Rollen, die ihre Visionen von urbaner Modernität in einer Serie von drei aufeinanderfolgenden Plänen festhielten. Diese Pläne strukturierten den städtischen Raum in funktionsräumlicher Gliederung mit klaren Gebietszuweisungen für Wohnen, Gewerbe, Grünflächen und Bildungseinrichtungen. Dabei wurden auch integrierte, mit sozialer Infrastruktur ausgestattete Stadtviertel in zentraler Lage ausgewiesen und teilweise fertiggestellt, bestehend aus in Fertigbauweise in sowjetischen Fabriken vorfabrizierten Häusern (sog. Micro-Rayons, Abb. 2 und 3).

Die drei Pläne aus den Jahren 1964, 1970 und 1978 integrierten weitestgehend die früheren, unter König Amanullah (1919-1929) durchgeführten Planungen, die von seinen Nachfolgern Nader Shah (1929-1933) und Zaher Shah (1933-1973) fortgeführt wurden und sukzessive in der Entstehung neuer Wohnviertel und neuer Verwaltungsgebiete mündeten, ebenso wie im Aufbau neuer Industrien und dazugehöriger Wohnviertel (Hahn 1972, Abb. 4). Dieser königliche „Traum der Modernisierung für ein neues und fortschrittliches Afghanistan“ (Schinasi 1917: 120-151) beruhte vornehmlich

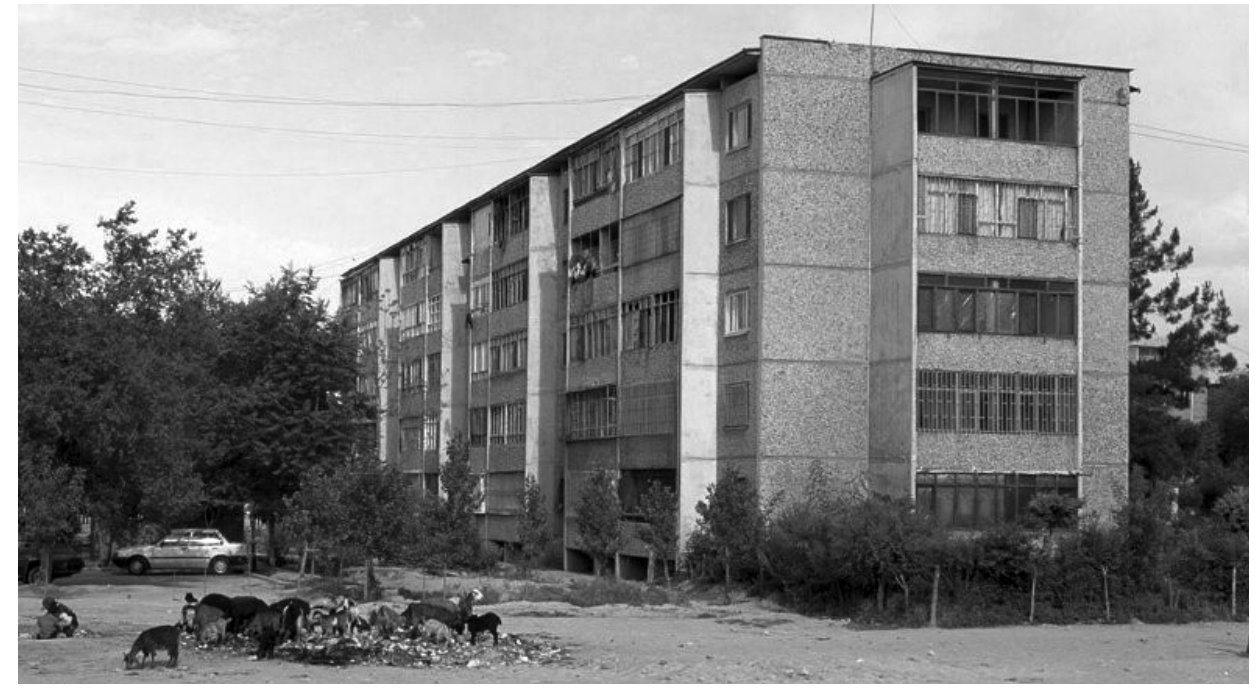

Abb. 2 Wohnblock in Microrayon 3 (Foto: Calogero 2003) 
Abb. 3 Kabul - Siedlungsgebiete und administrative Gliederung. Weitere bis 2017 eingemeindete Gebiete sind nicht auf der Karte markiert (Quelle: Calogero 2011)

Abb. 4 Zwischen 1923 und 1963 erbaute Stadtviertel von Kabul (Quelle: Calogero 2011)

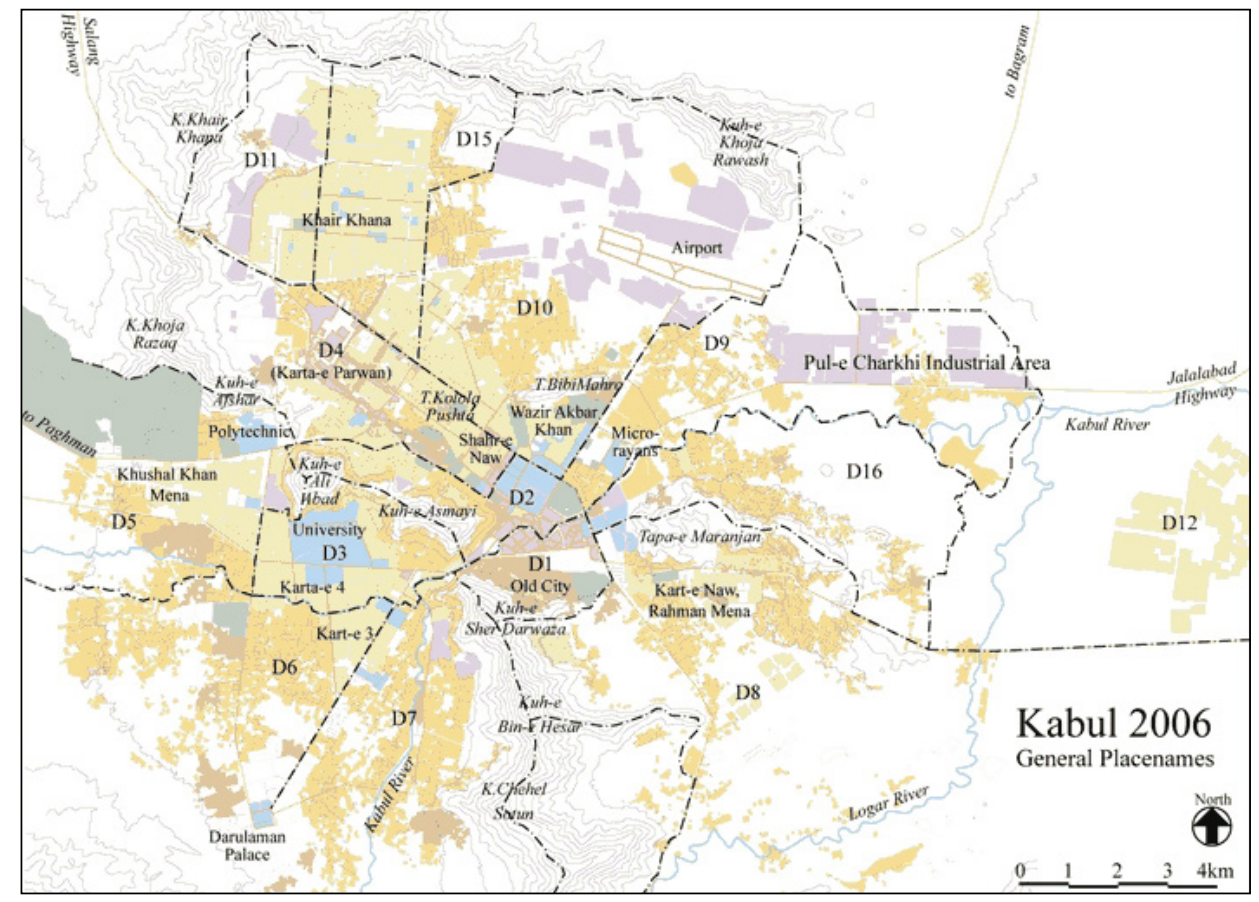

auf europäischer Expertise, und in den 1920er Jahren waren unter anderem der deutsche Architekt Walter Harten und ein Team von Ingenieuren federführend an der Neustrukturierung Kabuls beteiligt (ebd.).

Formell liegt die hauptstädtische Planungshoheit bei der Kabuler Stadtverwaltung, die heute direkt dem Staatspräsidenten unterstellt ist und den Status eines Ministeriums erhalten hat. In den 1930er Jahren wurde die Behörde zu einer funktionierenden Verwaltungseinrichtung geformt und pflegt seitdem ein Top-down-Planungsverständnis, in der die Direktiven städtischer Entwicklung von oben durchgesetzt werden. Auch während fast 40 Jahren Kriegszustand funktionierte die Behörde und verfügt bis heute über ein gutes institutionelles Gedächtnis. Nach der Vertreibung der Taliban

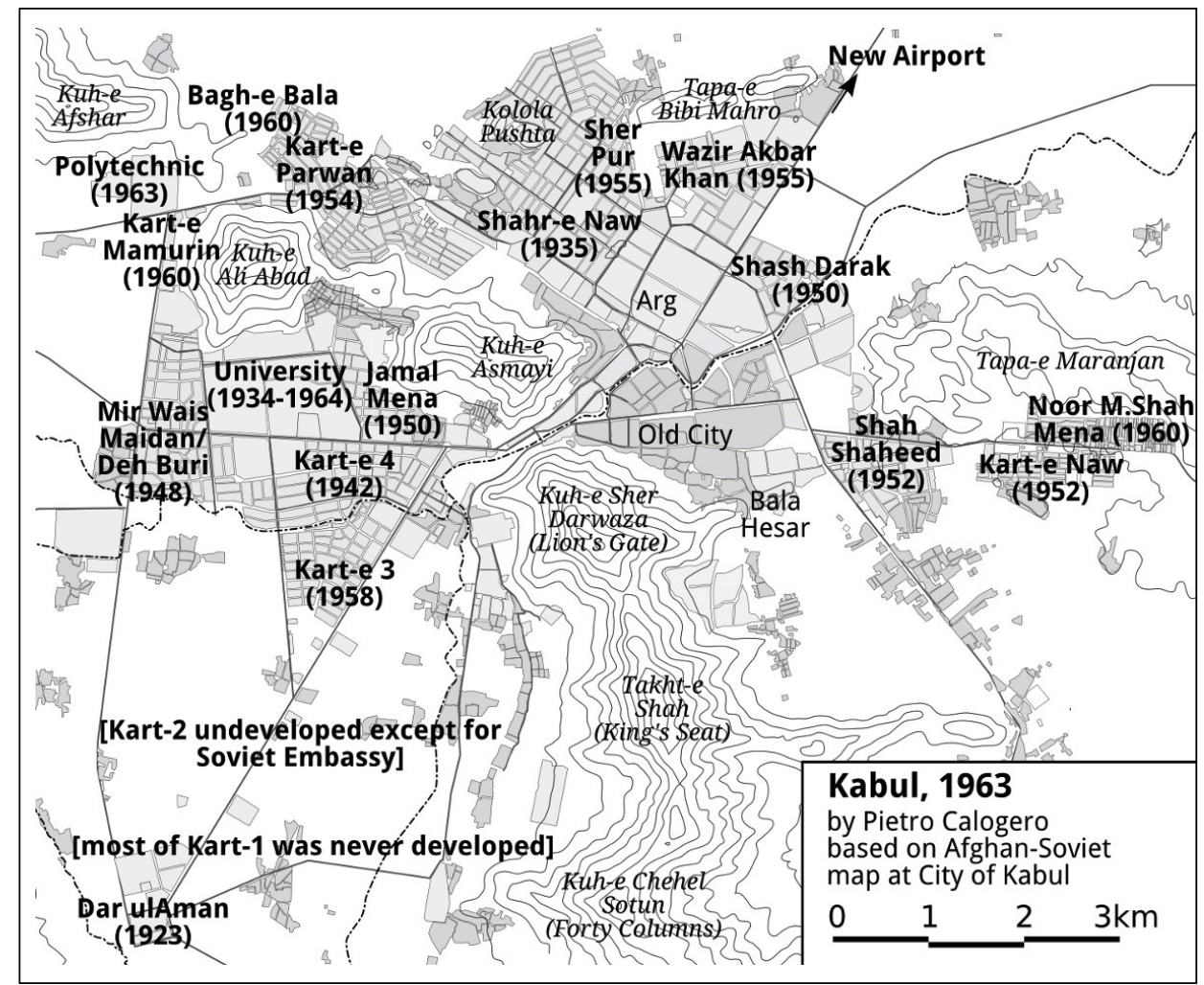


wurde im Jahre 2001 der letzte angefertigte Masterplan von 1978 wieder hervorgeholt und zur Steuerung künftiger Entwicklungen als maßgeblich bestimmt. Präsident Karzai erwirkte allerdings 2002 per Dekret eine Aussetzung der Implementation dieses Masterplans bis zur Erstellung einer zeitgemäßen Revision, die aktuelle Entwicklungen und Problemlagen berücksichtigt. Dennoch gilt ein funktionierender Masterplan weiterhin als das ultimative Instrument zur Steuerung der städtischen Entwicklung. Dies wird auch von dem seit 2001 wieder eigenständigen Ministerium für Stadtentwicklung und Wohnungsbau (MUDH) so gesehen. Das MUDH ist betraut mit städtischen Planungsaufgaben und Politikentwicklung in nationaler Perspektive, inklusive der Erstellung von Masterplänen für alle afghanischen Städte. In Bezug auf die Revision des Plans für Kabul hat die Stadtverwaltung einen Passus des noch unter den Taliban novellierten Kommunalrechts municipal law (Government of the Islamic Republic Afghanistan 2000) aktiviert und das Recht eingefordert, die alleinige Planungshoheit über Kabul zu erhalten.

MUHD und Stadtverwaltung befinden sich in steter Rivalität und ringen um die Kompetenz, stadtplanerische Prozesse in Kabul zu gestalten (Beall/ Esser 2005). Die besondere gesetzlich bestimmte Situation der Kabuler Stadtverwaltung und die Gleichstellung des Bürgermeisteramtes mit einem Ministerposten sind für diese Konkurrenz maßgeblich. Dabei ist die Stadtverwaltung grundsätzlich verantwortlich für die Umsetzung von geplanten Aufwertungsprozessen, Straßenbau, Wasser- und Abwasserversorgung und Abfallentsorgung. Hier wird das Anhängen am Masterplan besonders problematisch, denn viele dieser, wenn überhaupt nur sehr dürftig ausgeführten, Aufgaben beschränken sich auf die in den offiziellen Planungsgrundlagen spezifizierten Gebiete. Informelle Siedlungen als illegalisierte Raumkategorien werden hier per Definition von städtischen Dienstleistungen ausgeschlossen. Während die Stadtverwaltung für die Umsetzung von Planvorgaben zuständig ist, soll das MUDH diese Vorgaben im Rahmen einer nationalen Stadtentwicklungspolitik formulieren, aber ebenfalls einer praktischen Implementierung zuführen. In Kabul führen diese Überlappungen zu dem eher schwierigen Verhältnis beider Institutionen. Grundlegend für die Konkurrenz sind dabei auch unterschiedliche politische Rationalitäten auf Seiten der beteiligten Akteure in den Behörden. Ein großer Teil der Belegschaft der Stadtverwaltung von Kabul hat bereits unter technischer Assistenz sowjetischer Berater die tägliche Arbeit verrichtet und ist in einem entsprechenden Planungsverständnis geschult. Zudem hat die Behörde diverse Regimestürze nach 1973 überlebt. Die Entscheidungsebene des erst nach 2001 wieder neu formierten MUDH ist dagegen von Personen besetzt, die während der Kriegsjahre in westlichen Nationen Zuflucht gefunden haben und nach der Vertreibung der Taliban in hohen Positionen ein westliches Modell des Staatsaufbaus unterstützen. So war zum Beispiel der langjährige Vizeminister des MUDH zwischen 1984 und 1995 Mitarbeiter am Institut für Stadtbauwesen der RWTH Aachen.

Unumstritten in beiden Einrichtungen bleibt allerdings, dass Kabul einen Masterplan braucht - so wie es auch in Afghanistans municipal law gesetzlich festgeschrieben ist (Art. 5.1). Nun ist ein Masterplan vor allem eine Karte, eine graphische Repräsentation einer Vision von Stadtentwicklung aus der Perspektive modernistischer Stadtplaner. Dieser Funktionalismus versperrt 
Abb. 5 Planungskontinuum: Formal geplante Wohngebiete des Masterplans von 1978 sind in hellgrau dargestellt, informelle Wohngebiete in grau (Stand 2006) und überlappende Gebiete in dunkelgrau (Quelle: Calogero 2009). in seiner Verhaftung an Statuten allerdings einen Blick auf gelebte Realitäten in der Stadt. Entsprechend ist die unmittelbare Funktion des Masterplans die der Kontrolle, nicht die einer inklusiven oder an den Bedürfnissen der armen Bevölkerung orientierten Stadtentwicklung. Im Gegenteil, das Beharren auf Masterplanung schafft eine Situation, in der das, innen ' und ,außen ' definitorische Macht erhält. Städtische Entwicklungen, die außerhalb der Planvorgaben stattgefunden haben, werden illegalisiert; städtische Informalität wird sprichwörtlich produziert. Informalisierter städtischer Raum wird so durch den formalen Planungsprozess selber produziert (Abb. 5).

Die Weltbank hat im Jahre 2005 errechnet, dass 70 Prozent der Wohngebiete in Kabul aus informellen Siedlungen mit Lehmhäusern bestehen, die 80 Prozent der gesamten Stadtbevölkerung beherbergen (World Bank 2005). Dabei besteht in den meisten Fällen kein Zugang zu Trinkwasser, Sanitäreinrichtungen und Elektrizität. Diese Zahlen spiegeln eine definitorische Macht der masterplanbasierten Stadtplanung wieder, die aktuelle städtische Lebensrealitäten und die Folgewirkungen von fast 40 Jahren Krieg und Konflikt einfach unberücksichtigt lässt. In gewisser Weise wird hier die „dunkle Seite der Planung“ sichtbar, deren Praxis ganze Bevölkerungsgruppen systematisch ausgrenzt (Yiftachel 1995). Dabei wird zudem außer Acht gelassen, dass der enorme Bevölkerungsdruck in Kabul nur durch eben diesen informellen Siedlungsbau überhaupt aufgefangen werden konnte und zudem eine gewaltige kollektive Investition der Armen und Schutzsuchenden darstellt. Die diskursive Macht des Masterplans spiegelt sich auch im alltäglichen Sprachgebrauch der Bewohner von informellen Siedlungen in Kabul wieder, die bei der Beschreibung ihrer Lebenssituationen immer wieder darauf hinweisen, dass sie genötigt sind ,außerhalb der Karte ( gheyr-e naksha), das heißt des Masterplans, zu leben. Entsprechend werden informelle Siedlungen von ihrer Bewohnerschaft auch als ,zorabad' bezeichnet, was bedeutet: Gebiete die unter Missachtung gesetzlicher Vorgaben errichtet wurden.

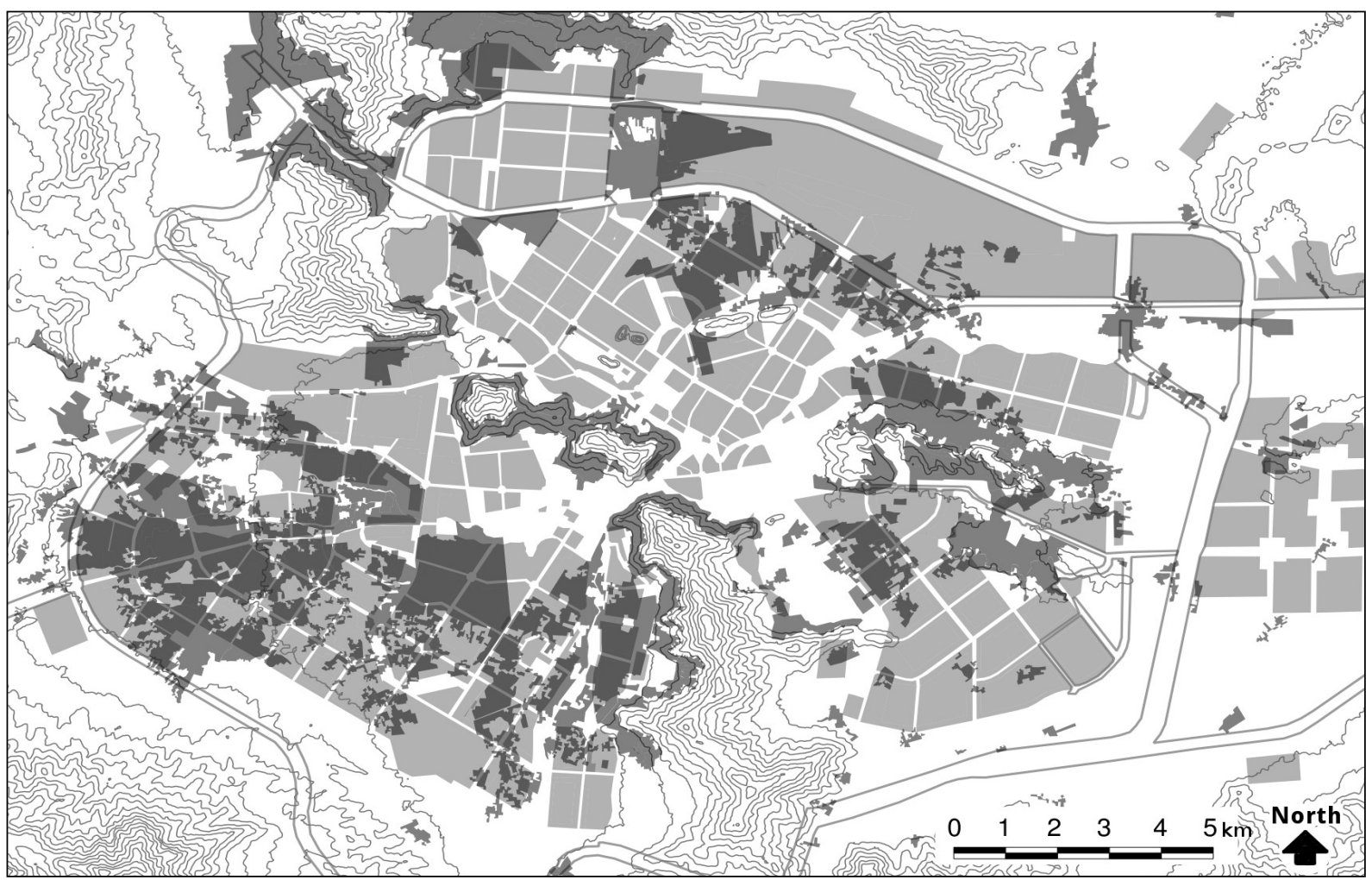


Während die Stadtverwaltung nun weiterhin keine Akzeptanz dieser städtischen Realität vorsieht, war das MUDH federführend mit an der Gestaltung der afghanischen Landpolitik (land policy) beteiligt (Government of the Islamic Republic Afghanistan 2007). In diesem Dokument wird ein differenzierter Umgang mit informellen Siedlungen angestrebt, der auf Aufwertung, Infrastrukturausbau und gesetzliche Legitimation ausgelegt ist und dabei auch die Problematik des städtischen Landraubs durch machtvolle Akteure thematisiert (Art. 2.2.4). Jenseits dieser Absichtserklärungen bleiben diese und weitere Aussagen des Dokuments bislang jedoch praktisch irrelevant und warten seit der Verabschiedung durch das Kabinett auf ihre Umsetzung. Als weiteres Beispiel der „Politik-Pantomime“ in Afghanistan (Blunt et al. 2017) reiht sich die Landpolitik damit in die Abfolge von ambitionierten Stadtentwicklungsprogrammen ein, die seit 2001 von im Wesentlichen westlichen Beratern entworfen wurden und nie eine richtige Umsetzung erfahren haben. Als additive „enzyklopädische Wunschlisten“, die sich am Zyklus der großen Geberkonferenzen zu Afghanistan orientieren und danach von Behörden und Geberinstitutionen gleichermaßen wieder vergessen werden (Leslie 2012), fällt eine realistische Prioritätensetzung schwer. Zudem wird die Frage nach der generellen Finanzierbarkeit ambitionierter Programme nicht beantwortet, so wie zum Beispiel im Kontext der, National Priority Programmes' (Government of the Islamic Republic Afghanistan 2012). Auch geht der internationale Fokus auf militärische Zielstellungen und die Bekämpfung aufständischer Gruppen stark zu Lasten der externen Unterstützung von Programmen der Stadtentwicklung.

Doch auch vor diesem schwierigen Hintergrund und großen Finanzierungslücken streben internationale Bemühungen im Einklang mit einem in afghanischen Stadtbehörden verankerten Modernisierungsverständnis große Lösungen an, um Kabuls vielfältige Problemlagen anzugehen. Derartige Überlegungen ignorieren jedoch weitestgehend, dass eine große Mehrheit der wachsenden Bevölkerung überhaupt keinen Zugang zu sozialer und physischer Infrastruktur hat, dass nur etwa fünf Prozent der städtischen Haushalte Zugang zu Abwassersystemen haben (Abb. 6), oder dass die hohe Aridität in Kabul zu starkem Staubaufkommen und in Verbund mit einem extremen und seit 2009 bereits verdoppeltem Verkehrsaufkommen zu außerordentlich gesundheitsgefährdender Luftverschmutzung führt (Integrated Regional Information Network 2008a, 2008b; UN Habitat 2016).

Exemplarisch für die angestrebten Großlösungen ist ein neuer Masterplan, der unterstützt durch die staatliche japanische Entwicklungsbehörde formuliert wurde und den Neubau eines neuen, modernen und ,eco-friendly' Kabul für drei Millionen Einwohner nordöstlich der bestehenden Stadtgrenzen vorsieht (Japan International Cooperation Agency 2011). Nach Worten eines hohen Beamten des MUDH soll das ,neue Kabul' nach westlichen Standards erbaut werden und Afghanistan in das 21. Jahrhundert überführen. In solchen Vorstellungen wird die komplette Abkopplung der Masterplanung von der sozialen und politischen Realität in Kabul sehr deutlich. Abgesehen davon ist das ländlich geprägte Gebiet für das neue Kabul mit Namen Deh Sabz (grünes Dorf) bereits von mehr als 50.00o Menschen bewohnt und es bestehen konkurrierende Gebietsansprüche zwischen der Stadtverwaltung und einem lokalen ,strongman'. Dies hat bereits zu Auseinandersetzungen 

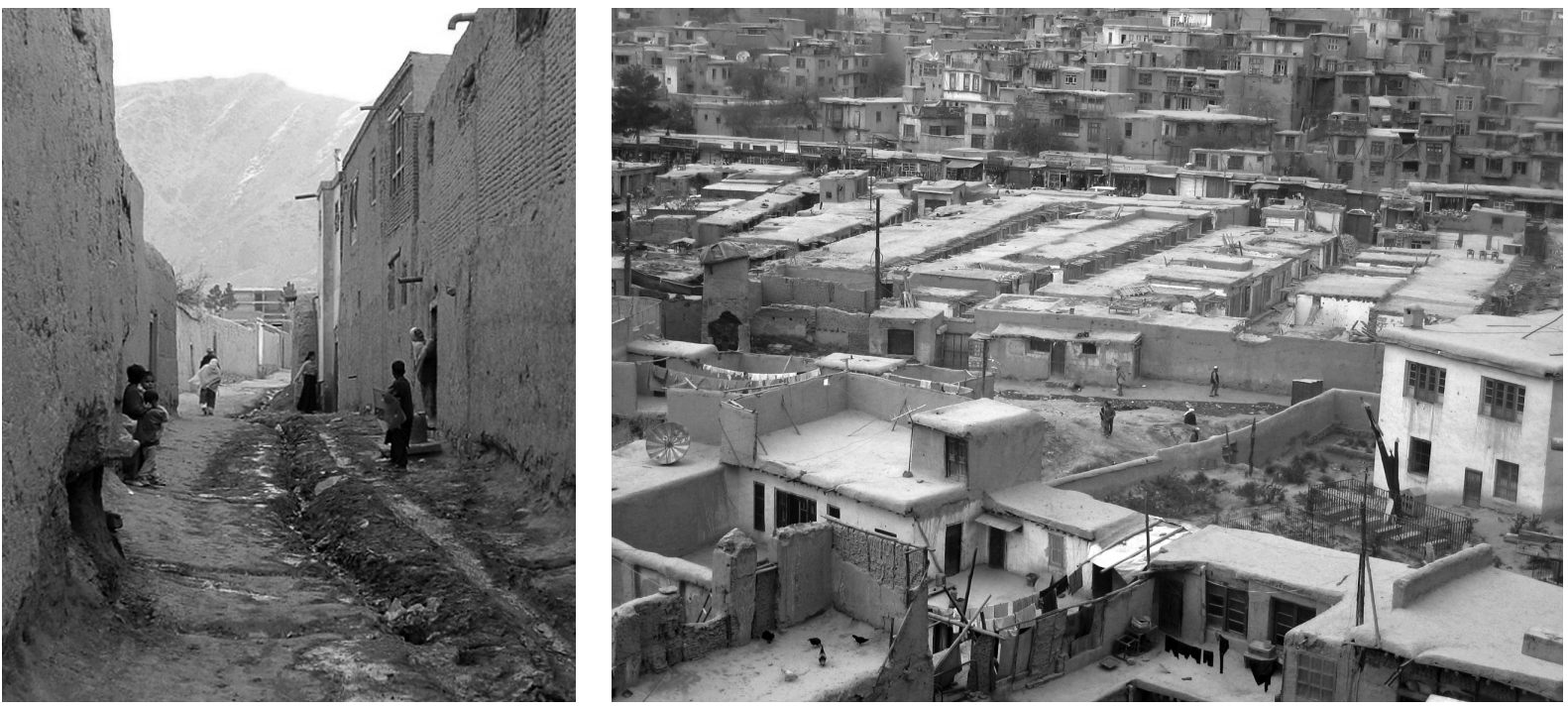

Abb. 6 Informelle Siedlung in Chehel Satun, Kabul. Abwasser wird in kleinen Entwässerungsgräben inmitten der Straße abgeleitet (Foto: Schütte 2005)

Abb. 7 Informelle Siedlung im innerstädtischen Kabul (Foto: Schütte 2005) und zuletzt 2013 auch zu Feuergefechten zwischen der Kabuler Polizei und Anhängern des lokalen Anführers geführt, die Todesopfer gefordert haben (Foschini 2013). Hier zeigt sich ein besonders deutliches Beispiel sowohl für übergeordnete Probleme von Landzugang, Landsicherheit und fehlender Rechtssicherheit in Landnutzungssystemen, die in ganz Afghanistan bestehen (Alden Wily 2003, Schütte 2015), als auch für die Problematik der Landnahme durch machtvolle Akteure, die entweder durch staatliche Institutionen sanktioniert sind - quasi als formale Informalität - oder die machtvoller als das staatliche Regime selber sind.

\section{Lehm als informelle Planung: Ausschluss und urbane Marginalität}

Informelle Planung repräsentiert einen relativ jungen Modus der Urbanisierung in Kabul. Erhebungen im Jahre 1976 zur Vorbereitung des Masterplans klassifizierten 10 Prozent der bebauten Fläche Kabuls als ,irregulär, und Interviews mit Angestellten der Stadtverwaltung, die noch unter der Führung des kommunistischen Regimes unter Najibullah (1987-1992) gearbeitet haben, weisen darauf hin, dass informelle Urbanisierung erst nach $1992 \mathrm{zu}$ einem wesentlichen Faktor der Stadtentwicklung geworden ist (Calogero 2011: 138). Im Spiegel formalisierter Stadtplanung, die unter den Leitlinien der Masterplanung Regelungsgewalt über diese Flächen beansprucht, wird hier Marginalität aktiv produziert und eine Ausweitung städtischer Dienstleistungen in diese Gebiete abgelehnt. Das MUDH hat unter der Ägide der Weltbank dagegen punktuell Aufwertungsaktivitäten in verschiedenen Siedlungen vorgenommen und verfolgt damit einen in westlicher Entwicklungsdiktion etablierten technokratischen Weg im Umgang mit urbaner Informalität.

Aufwertung ist aber nicht gleich Anerkennung, und der Gegensatz zwischen formaler Kohärenz und informaler Nonkonformität bleibt bestehen. Die Duldung informeller Urbanisierung durch ,Lehm' seitens städtischer Behörden ist jedoch ohne Alternative. In gewisser Weise zeigt diese Duldung auch die Informalisierung des städtischen Regimes selber, denn viele mit den städtischen Institutionen affiliierten Individuen profitieren auch in verschiedener Form von der zugewiesenen Extra-Legalität, zum Beispiel 
durch Bestechungen oder die Ansammlung später einlösbarer Gefälligkeiten (Calogero 2011: 147).

Der Typus ,Lehm' charakterisiert informelle Planung in Kabul, soll aber nicht darüber hinwegtäuschen, dass die oft in gegenseitiger Unterstützung erbauten Häuser solide und auch geordnete Strukturen bilden (Abb. 7). Es handelt sich hierbei tatsächlich um in hohem Maße intentional geplante Entwicklungen, die einen hohen Einsatz materieller Ressourcen verlangen. Entsprechend ist der Hausbau in neu auftauchenden Siedlungen ein sehr gradueller Prozess, der eine lange Zeit in Anspruch nehmen kann und im Wesentlichen von der Verfügbarkeit eines regelmäßigen Einkommens abhängig ist - eine im Kontext der Einbindung einer städtischen Mehrheit in , Regime informeller Sicherung ‘ äußerst seltene Qualität (Wood/Gough 2006, Schütte 2009). Daher erscheint es wenig verwunderlich, dass ein hoher Anteil der informellen Bevölkerung in Kabul nicht Hausbesitzer, sondern Mieter sind in zwei im Jahre 2003 untersuchten Siedlungen betrug dieser Anteil sogar 70 Prozent, wobei sich hier im Kontext der Rückkehr von Geflüchteten nach 2001 zwei oder mehrere Haushalte eine Wohnstatt teilen mussten (Calogero 2011, 154). Daraus ergeben sich teilweise extreme Bevölkerungsdichten (Abb. 8). Der Mietmarkt in Kabul ist zudem komplett ungeregelt, seit Karzai im Jahre 2004 das zuständige Ministerium für Wirtschaftsplanung aufgelöst hatte, um dessen Bemühungen einer Regulierung des sich ausbreitenden NGO-Sektors entgegenzutreten (ebd.: 27).

Kosten für Miete und Nahrung machten bei einer von Februar 2005 bis Januar 2006 durchgeführten longitudinalen Studie der Lebensbedingungen ausgewählter Haushalte in Kabul 82 Prozent des verfügbaren und stark schwankenden Monatseinkommens aus, und der verfügbare Rest musste für Gesundheits- und Heizkosten aufgewendet werden, während weitere Ausgaben über informelle Kreditbeziehungen finanziert sind, die zu dauerhaften Verschuldungssituationen führten (Schütte 2006a). Das alles geht einher mit einem enormen Aufwand für die Versorgung mit Trinkwasser und

Abb. 8 Bevölkerungsdichte pro Hektar in $50 \mathrm{im}$ Jahre 2003 untersuchten Siedlungen (Quelle: Calogero 2011)

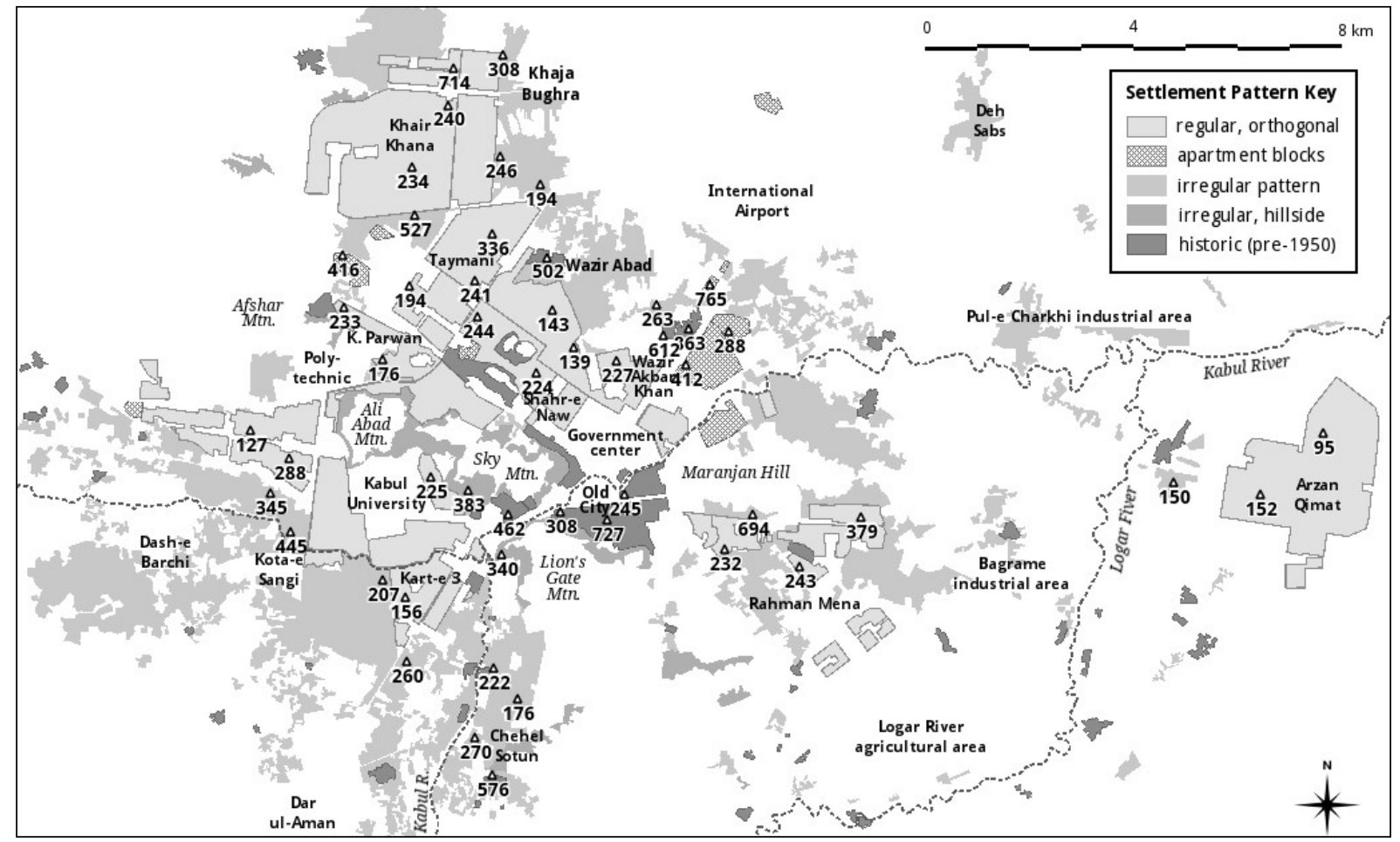


Abb. 9 Häuser in Breshna Kot im März 2005 (Foto: Schütte)

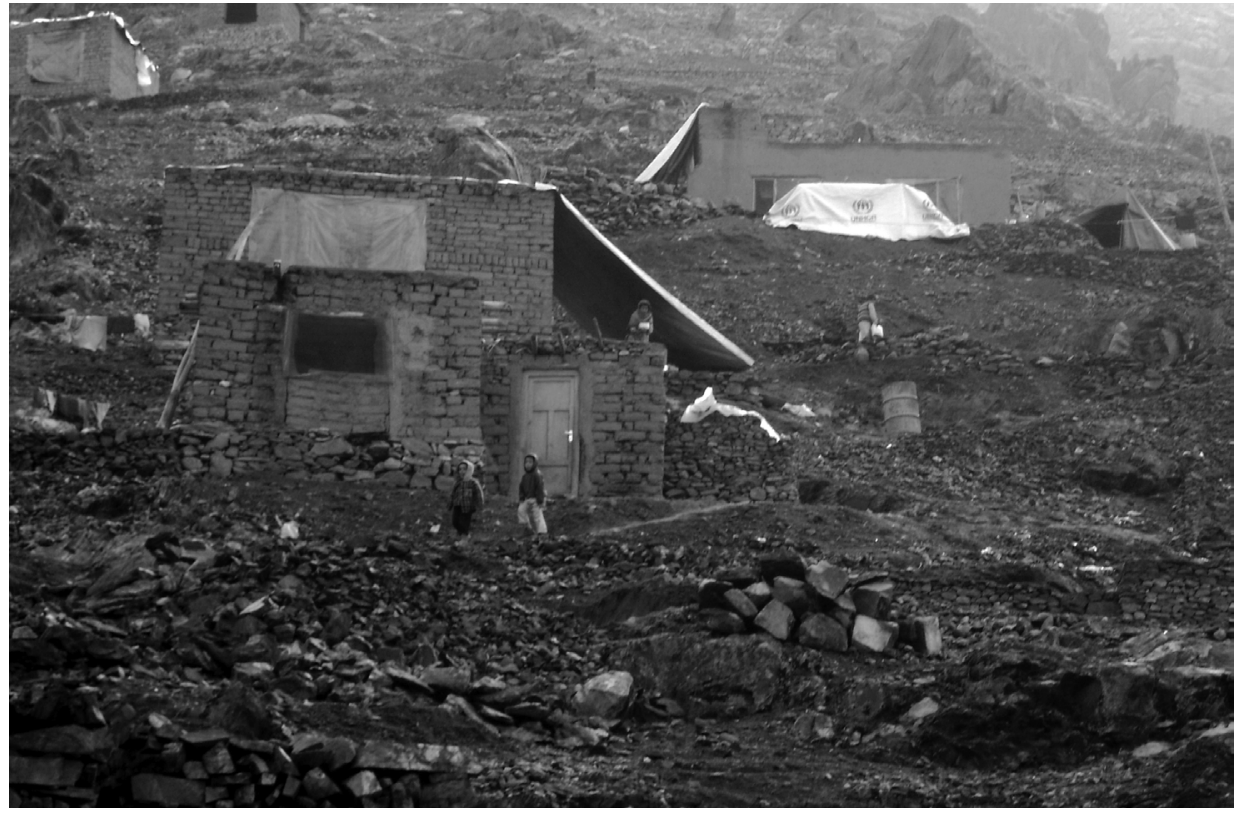

einer in den meisten informellen Siedlungen an der Oberfläche verlaufenden und stark gesundheitsgefährdenden Abwasserführung (Abb. 6). Siedlungen in Hanglage sind zudem gerade für ältere und gesundheitlich angeschlagene Menschen oftmals extrem schwer zugänglich.

Das Wachstum dieser Siedlungen ist dennoch ungebremst - Schätzungen von 2003 gingen noch von 6o Prozent der Siedlungsfläche von Kabul aus, 2007 waren es bereits 70 Prozent in einer nun auch flächenmäßig ausgedehnteren Stadt. Gerade an den Rändern Kabuls hat zudem in den letzten zehn Jahren ein stark erweiterter und zumeist ethnisch segregierter Siedlungsbau stattgefunden (Foschini 2017).

Die innenstadtnähere Siedlung mit Namen Breshna Kot im Südwesten von Kabul bietet dabei ein gutes Beispiel, um die bodenständigen Prozesse von Informalität als Modus der Urbanisierung besser zu verstehen. Breshna Kot wurde in Hanglage seit Herbst 2003 errichtet, abgesegnet von den Ministerialbeamten und ehemaligen Kriegsfürsten einer tadschikischen Faktion. Damit wurden die offiziellen Planvorgaben der Stadtverwaltung klar umgangen, um der nach ihrer Rückkehr aus Pakistan in Zeltlagern nahe des Kabuler Flughafens lebenden Gefolgschaft des Kriegsfürsten Bauland zur Verfügung zu stellen. Hier findet sich also eine durch offensive Landnahme gestützte Entwicklung, mit der sich machtvolle Akteure über Gesetz und Masterplan hinwegsetzen können, um sich damit die weitere Solidarität und möglicherweise auch monetäre Zahlungen ihrer Anhänger zu sichern. Derartige Prozesse städtischer Landnahme als Form von Patronage sind auch in anderen Städten Afghanistans zu beobachten (Schütte 2006b).

Die Möglichkeit, Land für den Hausbau erhalten zu können, sprach sich in den familiären Netzwerken im Aufnahmelager der Zurückgekehrten schnell herum. Auch einige aufmerksame Mieter im unterhalb der neuen Siedlung gelegenen Stadtteil Waisalabad nahmen die Gelegenheit wahr, sich ein Stück Land anzueignen. Auf diese Weise entstand binnen weniger Jahre eine neue Siedlung ohne jegliche infrastrukturelle Anbindung, in der die Häuser nach und nach und abhängig von der individuellen finanziellen Kapazität fertiggestellt wurden (Abb. 9). Für die Aussicht auf ein eigenes Haus nahmen die Menschen viele Strapazen auf sich - sie verkauften alles verzichtbare Hab 
und Gut zur Finanzierung der Baukosten, halfen sich dabei auch gegenseitig und bemühten ihre sozialen Netzwerke für finanzielle und professionelle Unterstützung. Zwar wissen alle, dass sie sich in Breshna Kot, außerhalb der Karte' befinden, doch hoffen die neuen Hausbesitzer, dass die bereits getätigten Investitionen sie vor einer möglichen Vertreibung schützen. Zu diesem Zweck hat sich auch ein Gemeindegremium gebildet, das sich nicht nur auf die Patronage des Kriegsfürsten verlassen möchte, sondern auch Petitionen an die Stadtverwaltung richtet, um Bleibezusagen zu erhalten und eine Versorgung mit Elektrizität einzufordern. Die Sorge um das Recht zu Bleiben ist hier essentiell, ebenso wie die Frage nach, lebhafter Infrastruktur' und der Kampf um Zugang zu essentiellen Dienstleistungen im Kontext von informeller Gemeinschaft, Solidarität und Bemühungen um Anerkennung (Amin 2014). Entwicklungspolitische Anstrengungen in diesem Bereich richteten sich zuletzt auf die Etablierung von städtischen „Community Development Councils“ und Stadtteilversammlungen für die Steuerung einer „Entwicklung von unten" (Government of the Islamic Republic Afghanistan 2015a), deren Einfluss ohne Zugriffsmöglichkeit auf ausreichende materielle Ressourcen aber beschränkt bleibt (Monsutti 2012).

Dieses und viele andere Beispiele zeigen, dass informelle Stadtentwicklung für eine große Mehrheit von Kabulis der einzig gangbare Weg ist. Dabei wird ein Zugang zu städtischen Ressourcen gesucht, der im Kontext Kabuls niemals unter Beachtung formaler Regelungen hergestellt werden könnte. Entsprechend bleiben die Spannungen zwischen den formalisierten Idealen des Masterplans und der unmittelbaren, informalisierten Realität für viele Menschen ein Quell großer Unsicherheit und Sorge. Hier wird besonders deutlich, wie eine große städtische Mehrheit durch das formale Regime und seine Weigerung der Ausweitung grundlegender Infrastruktur in Gebiete ,außerhalb des Plans“ sich selbst überlassen wird. Entwicklungspolitische Bemühungen um den Ausbau dringend notwendiger infrastruktureller Versorgung bleiben insular und zeigen keine allgemeine Wirkung. Realistisch einzuschätzende Vorschläge zur rechtsverbindlichen Regulierung und infrastrukturellen Versorgung aller Siedlungen in Kabul liegen seit langem vor, finden aber keine Umsetzung (World Bank 2005). Dazu bräuchte es sowohl eine deutlich höhere und besser kanalisierte finanzielle Unterstützung seitens internationaler Geber, als auch eine offizielle Anerkennung informeller Siedlungen im Sinne einer armutsorientierten Stadtentwicklung, wie sie in anderen Kontexten bereits Wirkung gezeigt hat (Budds et al. 2005).

Spiegelglas als exzeptionalistische Planung: Informalität von oben

Die dritte Dimension der Planung von Kabul betrifft den Nexus der Aneignungspraxis lokaler Eliten und die Bedürfnisse der internationalen Entwicklungsindustrie. Auch wenn, wie am Beispiel von Breshna Kot gezeigt, die Problematik der städtischen Landnahme auch im Rahmen einer Politik der Patronage zur Redistribution an eine von Armut und Ausgrenzung betroffene Bevölkerung erfolgen kann, so sind im Kontext der Informalität von oben noch weitere Aspekte bedeutsam.

Zwei Aspekte sind hier hervorzuheben: zum einen die Präsenz der internationalen Akteure aus Militär, Diplomatie und Entwicklungsindustrie und die dadurch hervorgerufenen Stadtentwicklungsprozesse, zum anderen die andauernden Landnahmen durch machtvolle Akteure - sowohl Kriegsfürsten 
und Kommandeure privater Milizen als auch Vertreter_innen der afghanischen Regierung -, die zunehmend die Stadtentwicklung in Kabul steuern. Vielfach sind die Grenzen zwischen Kriegsfürst und Regierungsvertreter jedoch als fließend zu begreifen, nämlich immer dann, wenn ein Zugriff auf durch offizielle Positionen vermittelte Ressourcen den Interessen machtvoller und über Privatarmeen verfügender Akteure dienlich sind (Giustozzi/ Orsini 2009, Mukhopadhyay 2014).

Der Terminus ,Spiegelglas 'verweist dabei einerseits auf die zunehmende Verwendung reflektierender Glaskonstruktionen in spekulativen Hochhauskonstruktionen, finanziert aus internationalem Kapital oder schattenwirtschaftlichen Betätigungen, die zum Beispiel als Shoppingcenter oder Luxushotels genutzt werden, - andererseits aber auch auf die Nutzung getönter Panzerglasscheiben in den SUVs der transnationalen Elite. In beiden Fällen signalisiert Spiegelglas Anonymität und Straffreiheit, aufrechterhalten durch Undurchsichtigkeit.

Die internationale Präsenz in Kabul hat insbesondere in den Anfangsjahren der Intervention enorme Ausmaße angenommen. So hat sich zum Beispiel die Anzahl in Kabul tätiger gemeinnütziger NGOs zwischen 2001 und 2005 mehr als verdreifacht und ist bis 2010 kontinuierlich weiter auf insgesamt 210 Organisationen angewachsen (Mitchell 2017). Hier sind die zahlreichen Geberinstitutionen, Vertretungen staatlicher Entwicklungsbehörden oder UN-Organisationen nicht einmal mitgerechnet. Von Beginn an basierte diese Präsenz auf räumlicher Segregation und der Trennung internationaler Entwicklungseinrichtungen vom normalen Alltag der Bevölkerung Kabuls. Die Entwicklungsblase beherbergte und sicherte die transnationale Elite, die ihr Bild der Stadt zu großen Teilen eher aus medial inszenierten Darstellungen und den Infrastrukturen des „war zone entertainments“ (Fluri/Lehr 2017: 36) als aus eigenen Ansichten und Begegnungen mit der Bevölkerung Kabuls gewonnen hat. Dies gilt noch viel mehr für das internationale Militär, dessen Angehörige ihren Einsatzalltag vornehmlich in ihren Garnisonen verbringen und für die das ,Draußen' ausschließlich feindlich ist. Dieses Primat der Sicherung hat zu exklusiven und stark geschützten Raumproduktionen geführt, die das Leben der Stadtbevölkerung eingrenzen und wesentlich zu den profanen, aber überbordenden Verkehrsproblemen in der Stadt beigetragen haben, indem zentrale Verkehrsachsen blockiert und damit unpassierbar gemacht wurden. Abbildung 10 illustriert die international gesteuerte Ausnahmeplanung in Kabul, die einer von Mauern aus Schanzkörben (HESCO-Walls) geschützten Befestigungsanlage gleicht und das gesamte in der Karte dargestellte Gebiet für die normale Stadtbevölkerung unzugänglich macht.

Weitere räumliche Transformationen betreffen die Beherbergungspolitik der internationalen Entwicklungsmaschinerie (Calogero 2011a). Eine Konzentration von Büroräumen und Unterkünften befindet sich dabei im wohlhabenderen und zentralen Stadtteil Wazir Akbar Khan, der im Jahre 1955 fertiggestellt wurde (s. Abb. 4; in Abb. 10 markiert als ,Wazir'). Dieser Stadtteil war aus verschiedenen Gründen attraktiv für den internationalen NGO-Sektor. Gelegen in der Nähe des Flughafens und der internationalen Botschaften und UN-Einrichtungen, bietet Wazir einen Bestand von großzügigen Häusern, in dem Büros und Unterkünfte für internationale Angestellte in Kombination geschaffen werden konnte. Die Grundstücke sind von hohen 


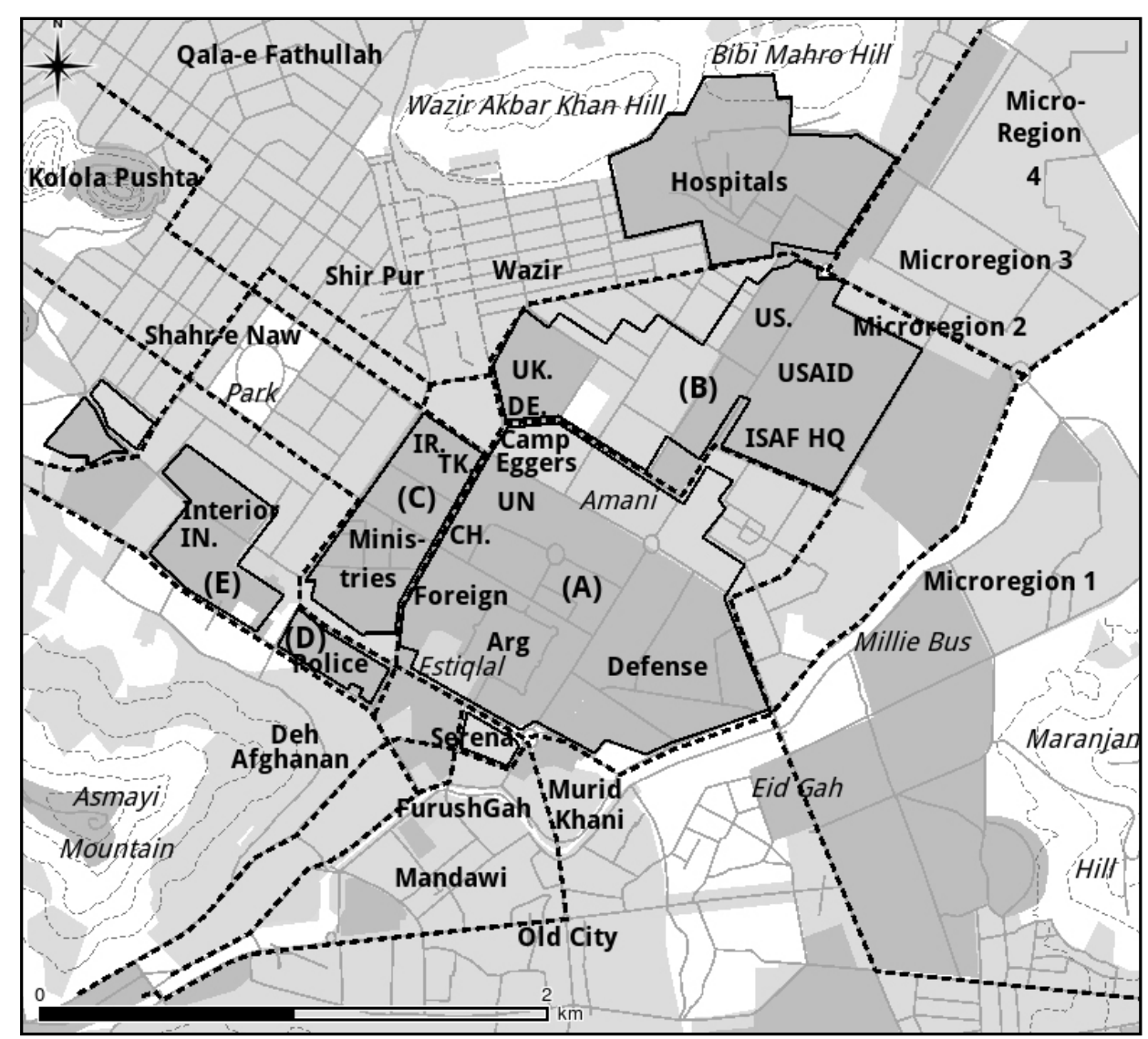

Mauern umgeben und wurden vielfach mit Stacheldraht und Schanzkörben abgesichert; sie sind groß genug, um unabhängige Infrastruktur in Form von Dieselgeneratoren für die Stromversorgung oder Satellitenschüsseln für Fernsehen und Internet bereitzustellen. Viele Hauseigentümer in Wazir konnten es sich leisten, Afghanistan während des Bürgerkriegs zu verlassen. Die Eigentumsrechte wurden dabei sowohl von den Mudschahedin als auch den Taliban aus Gründen der Zurückweisung des Staatssozialismus der 1980er und frühen 1990er Jahre akzeptiert, und nach der westlichen Invasion setzte die plötzliche, durch den Entwicklungssektor getragene, Gentrifizierung ein. Viele abwesende Hausbesitzer kündigten ihren langjährigen Mietern, um von den Entwicklungsorganisationen ein Vielfaches an monatlichen Mietzahlungen zu verlangen - Beträge von über 5.00o USD stellten dabei zu Beginn keine Ausnahme dar (Bearak 2002), mit weiterhin steigender Tendenz in den Jahren nach 2002. In Wazir und seiner durch den internationalen Sektor produzierten infrastrukturellen Autonomie lässt sich beinahe die Apotheose eines „splintering urbanism“ (Graham/Marvin 2001) wiederfinden, der einen hohen Druck auf den durch das rapide Bevölkerungswachstum ohnehin bereits strapazierten Mietmarkt in Kabul ausübte. Die Intervention sorgte für die Entstehung einer besitzenden Klasse von neuen Reichen und die Ausweitung der gesellschaftlichen Spaltung zwischen Arm und Reich. Aber auch die zuvor bereits wohlhabenden, machtvollen Akteure konnten sich weiter bereichern und die Richtung der städtischen Entwicklung entscheidend mitbestimmen.

Hier ist zunächst das vielbeachtete Beispiel von Sherpur zu nennen (Constable 2003, Nathan 2009, Calogero 2011) - ein ehemaliges, während des zweiten anglo-afghanischen Krieges im Jahre 1879 besetztes britisches Militärlager, das lange auch vornehmlich militärisch genutzt wurde und
Abb. 10 Die zentrale Innenstadt Kabuls und ihre internationale Architektur. Extrem gesicherte Gebiete sind dunkelgrau markiert, Straßenblockaden mit einer doppelt gestrichelten Linie und dauerhaft verstopfte Straßen einfach gestrichelt. Fünf Gebiete können hier unterschieden werden: (A) enthält den Präsidentenpalast, wichtige afghanische Ministerien, das Hauptquartier der UN-Mission in Afghanistan, die chinesische Botschaft und eine Militärgarnison. (B) beherbergt die Botschaften der USA, Großbritanniens, Deutschlands, Kanadas, Pakistan und Bulgarien sowie das ISAF-Hauptquartier und die Zentrale von USAID. Im Gebiet (C) befinden sich ein Ministerium und die Botschaften des Iran und der Türkei, in (D) das Hauptquartier der Kabuler Polizei und in (E) ein weiteres Ministerium (Quelle: Calogero 2007). 
offiziell dem afghanischen Verteidigungsministerium gehört. Hier in zentraler Lage hatte sich in den 1990er Jahren eine informelle Lehmsiedlung gebildet, die im September 2003 von polizeigeschützten Bulldozern planiert wurde, um Platz für Luxusbauten nach pakistanischem Vorbild zu schaffen (Abb. 10). Der einflussreiche tadschikische Verteidigungsminister Fahim zeigte sich verantwortlich und verteilte das nun freie Land an 29 einflussreiche Personen, viele davon in neuer Regierungsverantwortung unter westlicher Führung. Unter die neuen Landbesitzer gesellten sich der Bürgermeister von Kabul und seine Stellvertreter, der Stadtplanungsdirektor, verschiedene Minister der neuen Regierung, sowie einige Kommandeure und offenbar auch Verwandte aus dem Hause Karzai (Nathan 2009). Dies ist ein besonders offensichtliches Beispiel einer Informalität von oben, an der sich auch die Planungsbeauftragten und Vertreter der offiziellen, ,beton'-gestützten Stadtplanung großzügig bedienten. Gleichzeitig wurde hier schnell deutlich gemacht, wie die Kollaborateure und Profiteure der westlichen Intervention sich im ,neuen“ Afghanistan nach den Taliban ohne Konsequenzen über die formelle Gesetzeslage hinwegsetzen können. Hier werden auch praktische Widersprüche deutlich: Wenngleich offizielle Stadtplanungspolitik keine Umsetzung erfährt und in praktischer Hinsicht bedeutungslos bleibt und ein staatliches Gewaltmonopol nur auf dem Papier existiert, so werden im Sinne einer elitären Raumaneignung von größtenteils staatlichen Landflächen doch Fakten geschaffen, die einer Bereicherung und Machtzementierung von Wenigen dienen sollen.

Sherpur ist exemplarisch für diese Prozesse, aber nur ein frühes und vielbeachtetes Beispiel der elitären Landnahme zum Zwecke von Selbstbereicherung, Spekulation oder der Patronage (siehe auch Abb. 11). Es zeigt, dass Formalität und Gesetzestreue für die afghanische Elite keine praktische Funktion hat und für das Verfolgen eigener Interessen auch kein Hindernis darstellt. Die für Landnahmen verantwortlichen Akteure sind machtvoller als das städtische Regime und können auch von einer wenig einflussreichen Zentralregierung nicht in ihrem Handeln gestoppt werden.

Abb. 11 Die Nachbarschaft von Lehm und Spiegelglas: zwei informelle Häuser in Sherpur (Foto: Calogero 2007).

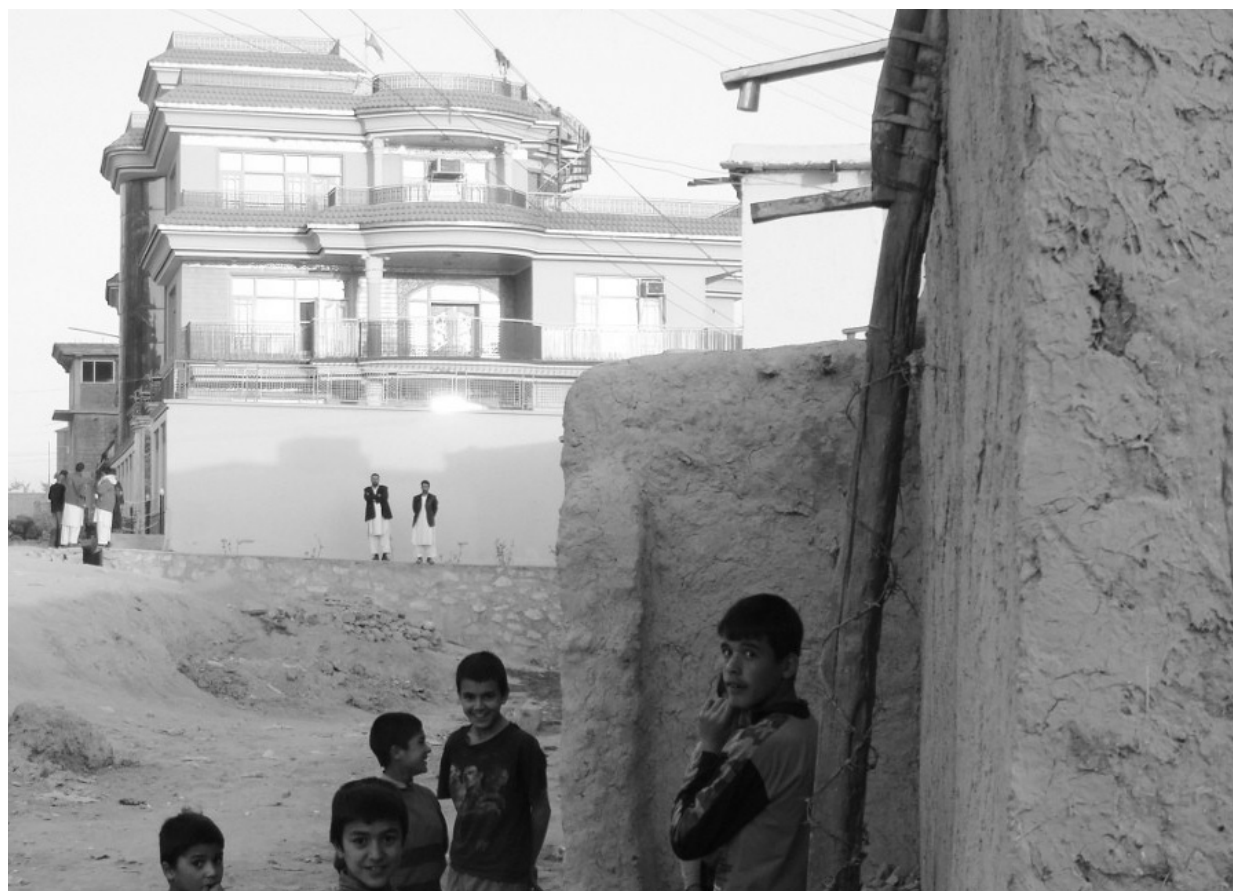


Heute nehmen auch die neuen, durch japanische Expertise beförderten Masterpläne in ihren Kartierungen von Kabul Bezug auf durch Kriegsfürsten und andere machtvolle Akteure „erbeutetes Land“(Japan International Cooperation Agency 2011). Dabei handelt es sich in sehr vielen Fällen um neue Wohngebiete (shahrak), die seit 2010 zunehmend die städtische Raumproduktion dominieren und aus kommerziellen Interessen heraus für wohlhabendere Schichten auf Staatsland gebaut werden (Abb. 12). Hier sind politische Akteure in offiziellen Ämtern besonders aktiv und nutzen ihre privilegierten Positionen, um äußerst lukrative Deals mit Geschäftsleuten und Investor_innen abzuschließen und von dem neuen Bauboom zu profitieren (Foschini 2017: 24).

In einen besonders prominenten Fall war mutmaßlich der damalige Vizepräsident und heutige Vorsitzende des „Hohen Friedensrates“ von Afghanistan, Karim Khalili, involviert (Pajhwok 2011). Es geht dabei um die politisch alimentierte Landnahme durch eine Baufirma und die Entwicklung einer äußerst profitablen shahrak auf einer im Masterplan als Grünfläche ausgewiesenen Parzelle. Auch der amtierende Bürgermeister von Kabul nutzte seine einflussreiche Position und wurde des Landraubs zur Beförderung des Baus von Hochhauswohnungen bezichtigt (Zaheer 2014). Afghanistans unabhängige Nachrichtenagentur Pajhwok hat noch einige weitere derartige Fälle der Informalität von oben dokumentiert, und es zeigt sich, dass sich im Rahmen der exzeptionalistischen Planung machtvolle Belange ausdrücken, die zunehmend die Stadtentwicklung von Kabul mitbestimmen. Land bildet dabei zusammen mit dem US-Dollar und Opium die bevorzugte Währung der Eliten, genutzt zur Ausübung von Kontrolle und Patronage. Die Appropriation von Land zum Bau vieler shahraks passiert fernab jeglicher Planvorgaben und gesetzlicher Bestimmungen und illustriert in deutlichster Weise die Informalität des Regimes selber in der Verfolgung persönlicher Interessen. Die 2009 neu gegründete afghanische Landbehörde ARAZI registriert diese Vorfälle und spricht von landesweit 355 illegal durch machtvolle Personen erbauten shahraks, doch bleibt diese Kenntnis wirkungslos im Angesicht von Akteuren, die stärker sind als das formale städtische Regime (8am Nachrichtenmagazin 2018). In Kabul selber befinden sich nach offiziellen Angaben insgesamt 38 dieser großflächig und vornehmlich von ehemaligen Kriegsfürsten und Mudschahedin errichteten Neubausiedlungen (ebd.).

Offizieller Siedlungsbau zur Unterstützung der Armutsbevölkerung ist dagegen fast gar nicht präsent. Es gibt zwei Projekte, die zur Unterstützung
Abb. 12 Moderne
Bauten in der Haji
Nabi shahrak im
Südwesten von Kabul
(Foto: Elham Gharji
2018)

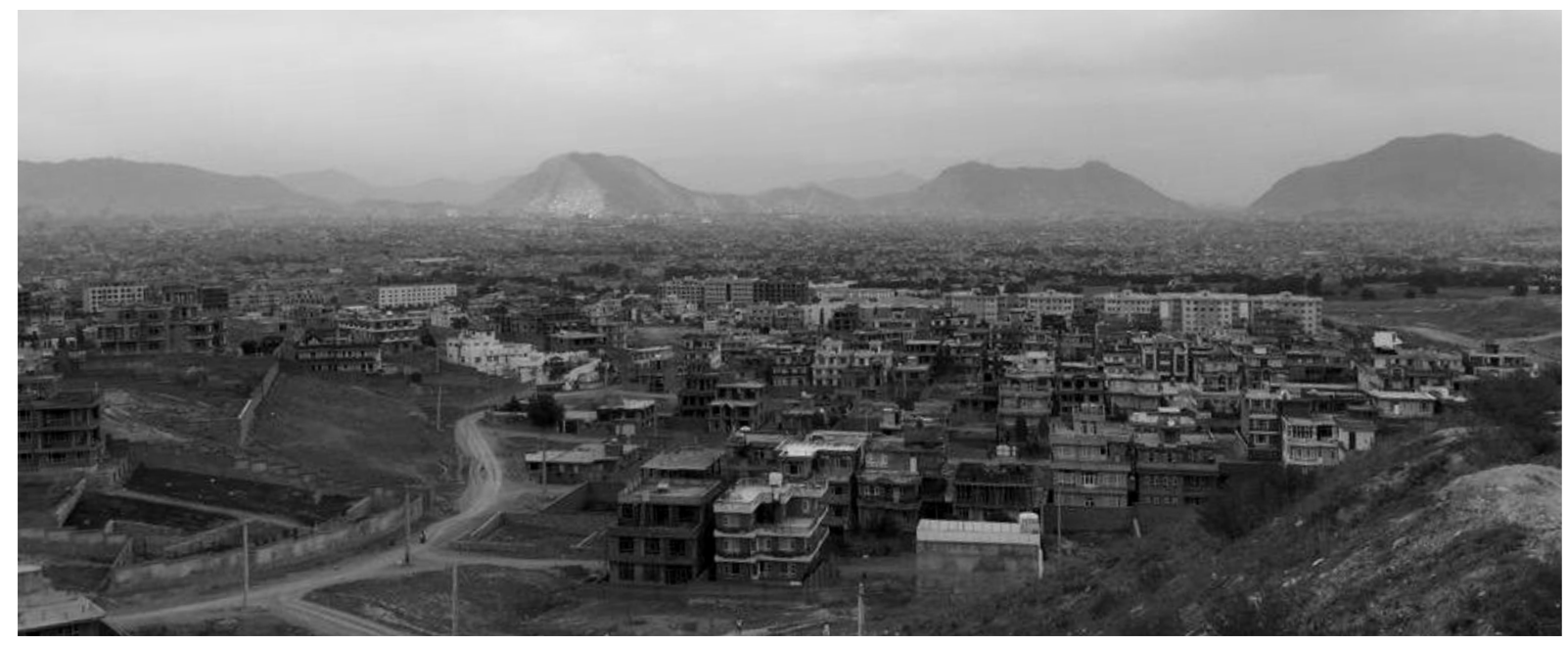


zurückgekehrter Geflüchteter unter ministerialer Kontrolle umgesetzt wurden. Diese befinden sich jedoch auf kommerziell uninteressanten Flächen, mehr als 30 Kilometer vom Stadtzentrum entfernt ohne Straßenanbindung, Einrichtungen der Grundversorgung oder Einkommensmöglichkeiten für die dorthin umgesiedelten Haushalte (Foschini 2017: 23f.). Hier wird erneut deutlich, wie interessensgesteuerte Stadtentwicklung an den Bedürfnissen einer Mehrheit vorbeigeht und die Appropriation öffentlichen Landes durch machtvolle Akteure von staatlichen Behörden nicht verhindert werden kann. Elitäre Interessen setzen sich durch, und der Staat und seine Institutionen sind in praktischer Hinsicht fast bedeutungslos. Ob die neuen, von internationalen Gebern organisierten Programme mit Blick auf die Verbesserung städtischen Managements dies ändern können, darf bezweifelt werden (Kammeier/Issa 2017).

DiebestehendenunddurchalltäglichePraxisgefestigten Machtasymmetrien erlauben eine an der armen Bevölkerung orientierte Stadtentwicklung nur zu Zwecken der Patronage von Gefolgsleuten. In weiterer Hinsicht zeigt sich hier aber auch das Versagen des westlichen Staatsaufbauprojektes in Afghanistan, das weiter zunehmend seine Legitimität verliert. Nicht zuletzt durch die Abkehr vom westlichen Projekt nehmen die kriegerischen Konflikte im ganzen Land deutlich zu (European Asylum Support Office 2017) und lassen den Alltag in Kabul zunehmend riskanter werden, wie auch die jüngste Anschlagsserie im Januar 2018 verdeutlicht.

\section{Die Normalität informeller Stadtentwicklung}

In ihrer Verknüpfung zeigen die hier exemplifizierten Modi der Stadtplanung von Kabul vor allem, dass die Orientierung an Planvorgaben oder gesetzlichen Regelungen eher die Ausnahme darstellt. Die Modi von Lehm und Spiegelglas bilden in ihrem ungleichen Zusammenspiel die Basis der Stadtentwicklung. Entsprechend lässt sich im Sinne Ananya Roys (2011) ganz klar von Informalität als „Idiom der Urbanisierung“ sprechen. Die „Dekonstruktion staatlicher Legitimität und ihrer Instrumente“ (ebd.: 233, Übers. d. A.) wird im Kontext von Kabul offensichtlich, jedoch mit sehr unterschiedlichen Konsequenzen für die Beteiligten einer Informalität von unten oder von oben. Im ersteren Fall wird durch die formellen Akteure der Stadtplanung und Stadtentwicklung eine illegalisierte und abhängige Bevölkerung produziert, die zwar geduldet, aber nicht erwünscht ist und entsprechend in der Breite nicht mit zentraler Infrastruktur versorgt wird. Dies ist die Perspektive, die von Entwicklungsorganisationen und Entwicklungsforschung mit informeller Urbanisierung als räumlicher Kategorie und Organisationsform verknüpft wird. Hier geht es dann entwicklungspraktisch um die Möglichkeit, Aufwertungsprozesse durchzuführen und Landrechte einzufordern, um Anerkennung zu garantieren, und in der Forschung um die Analyse von Strategien und Prozessen, die ein städtisches Überleben im Kontext von Ausgrenzung und Benachteiligung ermöglichen. Auch der Bezug zur progressiven Inwertsetzung einer in afghanischen Behörden durchaus bestehenden Expertise in technokratischen Planungsfragen wird im Rahmen anwendungsorientierter Forschung relevant. 
Im zweiten Fall sind die formellen Akteure selber federführend an der Produktion von Informalität beteiligt und profitieren dabei von exzeptionalistischer Planung. Dies ist eine Perspektive, die erst in jüngerer Zeit entwickelt wurde und die von oben gesteuerte informelle Stadtentwicklung als einen im städtischen Regime selber verankerten Prozess identifiziert (Roy 2003). Wenn urbane Herrschaft selber Informalität produziert, dann werden diese Prozesse auch herrschaftlich gesteuert: Die Politik von Missachtung und Vernachlässigung, von dauerhafter Verwundbarkeit und Armut, von Korruption und Bereicherung ist dementsprechend ebenso geplant wie zum Beispiel der Bau von Straßen oder Plätzen. Politische Herrschaft ist damit ebenso verantwortlich für die Produktion einer mittellosen städtischen und illegalisierten Mehrheit wie für die eigene vorsätzliche und bereitwillige Übertretung von Gesetzen und Regularien in dem sicheren Bewusstsein, dies gefahrlos tun zu können.

Der Blick nach oben ist für ein Verständnis von Prozessen globaler Urbanisierung unausweichlich, und elitäre Informalität bildet im Kontext der räumlichen Formationen und Transformationen von Kabul einen entscheidenden Faktor. Mit Blick auf urbane Restrukturierungen im Kontext von Staatsaufbau und Konflikt bietet das Beispiel Kabuls und seine durch verschiedene Planungsregime gesteuerte städtische Entwicklung Einblicke in die Modi machtgesteuerter Wohlstandsvermehrung, die in kalkulierter Weise die Schwäche des städtischen Regimes ausnutzt. Die in den beschriebenen Raumproduktionen materialisierten städtischen Machtverhältnisse verhindern die ohnehin äußerst rar gesäten Bemühungen um armutsorientierte Stadtentwicklung und politische Inklusion einer städtischen Mehrheit. Dominante Machtverhältnisse jenseits staatlicher Sanktionskompetenz regulieren die städtische Entwicklung und sorgen für einen explodierenden Bodenmarkt. Eine internationale Geberpolitik, die geleitet wird vom Glauben an die Gleichsetzung von städtischer ökonomischer Entwicklung mit städtischer Sicherheit, ist direkt an einer Zementierung der Stadtentwicklung von oben in Kabul beteiligt. Damit ist Kabul allerdings nicht alleine, und Beispiele aus anderen städtischen Konfliktregionen zeigen ebenfalls, wie städtische Bodenmärkte als Vehikel für die Akkumulation privaten Reichtums im Kontext von sogenannten Friedensmissionen dienen (Esser 2013). Die Auffassung kalkulierter urbaner Informalität als hybride Praxis und machtvolles strategisches Werkzeug ist entsprechend geeignet, die Ambiguität stadtpolitischer und -planerischer Entwicklungen in Kabul und anderswo besser zu begreifen und in übergeordnete globalpolitische Zusammenhänge einzuordnen.

Die Publikation dieses Beitrags wurde durch den DFG geförderten OpenAccess Publikationsfonds der FU Berlin ermöglicht.

\section{Autor_innen}

Pietro Calogero; Stadtplaner; Arbeitsschwerpunkte sind Stadtplanung und Planungstheorie, Globale Urbanisierung, Urbane Regime und Critical Modernity Studies.

pietro@calogero.us 
Stefan Schütte; Geograph mit Schwerpunkt Geographische Entwicklungsforschung; regionale Expertise in Südasien und Afghanistan zu Themen städtischer Lebenssicherung und ländlicher Entwicklung.

stefan.schuette@fu-berlin.de

\section{Literatur}

8am Nachrichtenmagazin (2018): Shahraks Under the Magnifying Glass - Bericht des online Nachrichtenmagazins vom 18. Februar. www.8am.af/x8am/1396/11/29/areas-underthe-magnifying-glass/ (letzter Zugriff am 5.9.2018, übersetzt von Sayed Mahdi Mosawi).

Alden Wily, Liz (2003): Land Rights in Crisis. Restoring Tenure Security in Afghanistan. Kabul: Afghanistan Research and Evaluation Unit.

AlSayyad, Nezar (2004): Urban informality as a „new“ way of life. In: Ananya Roy / Nezar AlSayyad (Hg.): Urban Informality: Transnational Perspectives From the Middle East, Latin America, and South Asia. Maryland: Lexington Books, 7-30.

Altrock, Uwe (2012): Conceptualising informality: Some thoughts on the way towards generalisation. In: Colin McFarlane / Michael Waibel (Hg.): Urban Informalities. Reflections on the Formal and Informal. Aldershot: Routledge, 171-193.

Amin, Ash (2014): Lively infrastructure. In: Theory, Culture \& Society 31/7-8: 137-161.

Bayat, Asef (2007): Radical religion and the habitus of the dispossessed: Does Islamic militancy have an urban ecology? In: International Journal of Urban and Regional Research 31/3: 579-590.

Beall, Jo / Esser, Daniel (2005): Shaping Urban Futures: Challenges to Governing and Managing Afghan Cities. Kabul: Afghanistan Research and Evaluation Unit.

Beall, Jo / Schütte, Stefan (2006): Urban Livelihoods in Afghanistan. Kabul: Afghanistan Research and Evaluation Unit.

Bearak, Barry (2002): Kabul journal: in the Afghan capital, rents go through the roof. In: New York Times, May 14.

Bechhoefer, William B. (1977): The role of squatter housing in the urbanization of Kabul. In: Afghanistan Journal 4/1: 3-8.

Beyer, Elke (2012): Competitive coexistence: Soviet town planning and housing projects in Kabul in the 1960s. In: The Journal of Architecture 17/3: 309-332.

Blunt, Peter / Mamundzay, Farid / Nasary, Muqtader (2017): The long and the short of policy pantomime in Afghanistan. In: Progress in Development Studies 17/1: 67-88.

Budds, Jessica / Teixeira, Paulo / SEHAB (2005): Ensuring the right to the city: pro-poor housing, urban development and tenure legalization in São Paulo, Brazil. In: Environment and Urbanization 17/1: 89-114.

Calogero, Pietro Anders (2011): Kabul cosmopolitan: geopolitical empire from the planner's viewpoint. In: Planning Theory 10 (1): 66-78.

Calogero, Pietro Anders (2011a): Planning Kabul: The Politics of Urbanization in Afghanistan. Berkeley: University of California, Department of City and Regional Planning, PhD-Thesis.

Constable, Pamela (2003): Land grab in Kabul embarrasses government / Mud homes razed to make room for top Afghan officials. In: Washington Post am 16.9.2003.

Cullather, Nick (2002): Damming Afghanistan: Modernization in a buffer state. In: The Journal of American History 89/2: 512-537.

Daulatzai, Anila (2008): The discursive occupation of Afghanistan. In: British Journal of Middle Eastern Studies 35/3: 419-435.

Davis, Mike (2006): Planet of Slums. London/New York: Verso.

Dörre, Andrei / Kraudzun, Tobias (2012): Persistence and change in Soviet and Russian relations with Afghanistan. In: Central Asian Survey 31/4: 425-443.

Dorronsoro, Gilles (2000): Revolution Unending. Afghanistan: 1979 to the Present. London: Hurst.

Dorronsoro, Gilles (2007): Kabul at War (1992-1996): State, ethnicity and social classes. In: South Asia Multidisciplinary Academic Journal. http://samaj.revues.org/document212. html (letzter Zugriff am 24.9.2018).

Esser, Daniel (2013): The Political Economy of Post-invasion Kabul, Afghanistan: Urban Restructuring beyond the North-South Divide. In: Urban Studies 50/15: 3084-3098. 
European Asylum Support Office (2017): EASO Country of Origin Information Report. Afghanistan Security Situation. Brussels: EASO.

Fluri, Jennifer L. / Lehr, Rachel (2017): The Carpetbaggers of Kabul and Other AmericanAfghan Entanglements. Intimate Development, Geopolitics, and the Currency of Gender and Grief. Athens: University of Georgia Press.

Foschini, Fabrizio (2013): Land Grabs (2): Deh Sabz, the new and the old. Kabul: Afghanistan Analysts Network.

Foschini, Fabrizio (2017): Kabul and the Challenge of dwindling Foreign Aid. Washington D.C.: United States Institute of Peace.

Giustozzi, Antonio (2009): Empires of Mud. Wars and Warlords in Afghanistan. London: Hurst.

Giustozzi, Antonio / Orsini, Dominique (2009): Centre-periphery relations in Afghanistan: Badakhshan between patrimonialism and institution-building. In: Central Asian Survey 28/1: 1-16.

Government of the Islamic Republic Afghanistan (2000): Municipal Law of Afghanistan 1379 (2000). Kabul: Ministry of Justice.

Government of the Islamic Republic Afghanistan (2007): Land Policy of Afghanistan. Kabul: Islamic Republic of Afghanistan.

Government of the Islamic Republic Afghanistan (2012): Urban Management Support Programme. Kabul: National Priority Programmes, Infrastructure Development Cluster.

Government of the Islamic Republic Afghanistan (2015): Estimated Population of Kabul City by District and Sex 2015-16. Kabul: Central Statistics Organization.

Government of the Islamic Republic Afghanistan (2015a): The State of Afghan cities 2015. Kabul: GoIRA.

Graham, Stephen / Marvin, Simon (2001): Splintering Urbanism: Networked Infrastructures, Technological Mobilities, and the Urban Condition. New York: Routledge.

Gregorian, Vartan (1969): The Emergence of Modern Afghanistan. Politics of Reform and Modernization, 1880-1946. Stanford: Stanford University Press.

Hahn, Helmut (1972): Wachstumsabläufe in einer orientalischen Stadt am Beispiel von Kabul/Afghanistan. In: Erdkunde 26/1: 16-33.

Integrated Regional Information Network (2008a): Afghanistan: Poor sanitation, bad toilets cause deaths, misery. UN Office for the Coordination of Humanitarian Affairs.

Integrated Regional Information Network (2008b): Kabul's air pollution putting people's health at risk. UN Office for the Coordination of Humanitarian Affairs.

Japan International Cooperation Agency (2011): Draft Kabul City Master Plan. Product of Technical Cooperation Project for Promotion of Kabul Metropolitan Area Development. Sub Project to Revise the Kabul City Master Plan. Kabul: JICA u. a.

Kammeier, Detlef H. / Issa, Zabihullah (2017): Urban Governance in Afghanistan: Assessing the New Urban Development Programme and Its Implementation. Kabul: Afghanistan Research and Evaluation Unit.

Kogan, Felix (2002): World droughts in the new millennium from AVHRR-based vegetation health indices. In: EOS, Transactions American Geophysical Union. 83/48: 557-564.

Kreutzmann, Hermann / Schütte, Stefan (2010): Persistence and change of Afghan-German economic cooperation - The case of the New Baghlan Sugar Company. In: Erdkunde 64/1: 1-16.

Kühn, Florian B. (2014): „We are all in this together ...“: Deutschland in der Ambiguität der Afghanistanintervention. In: Michael Daxner (Hg.): Deutschland in Afghanistan. Oldenburg, 193-211.

Leslie, Jolyon (2012): Urban recovery, or chaos? In: Martine van Bijlert / Sari Kouvo (Hg.): Snapshots of an Intervention. The Unlearned Lessons of Afghanistan's Decade of Assistance (2001-11). Kabul: Afghanistan Analysts Network, 125-129.

McFarlane, Colin / Waibel, Michael (2012): Introduction: The informal-formal divide in context. In: Colin McFarlane / Michael Waibel (Hg.): Urban Informalities. Reflections on the Formal and Informal. Aldershot: Routledge, 1-12.

Misdaq, Nabi (2006): Afghanistan. Political frailty and external interference. London/New York: Routledge.

Mitchell, David F. (2017): NGO presence and activity in Afghanistan, 2000-2014: A provincial-level dataset. In: Stability: International Journal of Security and Development 6/1: Art. 5, 1-18 
Monsutti, Alessandro (2012): Fuzzy sovereignty: rural reconstruction in Afghanistan, between democracy promotion and power games. In: Comparative Studies in Society and History 54/3: 563-591.

Mukhopadhyay, Dipali (2014): Warlords, Strongman Governors, and the State in Afghanistan. Cambridge: Cambridge University Press.

Nathan, Joanna (2009): Land grab in Sherpur: monuments to powerlessness, impunity, and inaction. In: Middle East Institute (Hg.): Afghanistan, 1979-2009: In the Grip of Conflict. Washington, D.C.: Middle East Institute, 71-74.

Ostermeier, Lars (2017): Imaginationen rechtsstaatlicher und demokratischer Polizei. Deutsche Polizeiprojekte in Afghanistan von 1957 bis 2010. Weinheim: Beltz Juventa.

Pajhwok Afghan News (2011): Khalili denies playing any role in land-grab by Onyx. https:// www.pajhwok.com/en/2011/04/21/khalili-denies-playing-any-role-land-grab-onyx (letzter Zugriff am 24.8.2018).

Poullada, Leon B. (1973): Reform and Rebellion in Afghanistan. King Amanullah's failure to modernize a Tribal Society. Ithaca/London: Cornell University Press.

Roy, Ananya (2003): City Requiem, Calcutta. Gender and the Politics of Poverty. Minneapolis. Minneapolis: University of Minnesota Press.

Roy, Ananya (2005): Urban informality. Toward an epistemology of planning. In: Journal of the American Planning Association 71/2: 147-158.

Roy, Ananya (2009): Why India cannot plan its cities: informality, insurgence and the idiom of urbanization. In: Planning Theory 8/1: 76-87.

Roy, Ananya (2011): Slumdog cities: rethinking subaltern urbanism. In: International Journal of Urban and Regional Research 35/2: 223-238.

Schinasi, May (2017): Kabul: A History 1773-1948. Leiden: Brill.

Schütte, Stefan (2006a): Searching for Security: Urban Livelihoods in Kabul. Kabul: Afghanistan Research and Evaluation Unit.

Schütte, Stefan (2006b): Poor, Poorer, Poorest: Urban Livelihoods and Vulnerability in Mazar-i-Sharif. Kabul: Afghanistan Research and Evaluation Unit.

Schütte, Stefan (2009): Informal (in)security in urban Afghanistan. In: Iranian Studies 42/3: 465-491.

Schütte, Stefan (2015): Peacebuilding and pasture relations in Afghanistan. In: Peacebuilding 3/3: 238-260.

Suhrke, Astri (2011): When More Is Less. The International Project in Afghanistan. New York: Columbia University Press.

Turton, David / Marsden, Peter (2002): Taking Refugees for a Ride? The politics of refugee return to Afghanistan. Kabul: Afghanistan Research and Evaluation Unit.

UN Habitat (2006): State of the World's Cities Report 2006/2007. 30 Years of Shaping the Habitat Agenda. London: Earthscan.

UN Habitat (2016): On The Move in Kabul City Region. Transportation and mobility for effective city region functioning and prosperity. Fukuoka: UN Habitat Regional Office for Asia and the Pacific.

Wood, Geof / Gough, Ian (2006): A comparative welfare regime approach to global social policy. In: World Development 34/10: 1696-1712.

World Bank (2005): Kabul: Urban Land in Crisis. Washington, D.C.: World Bank.

Yiftachel, Oren (1995): The dark side of modernism: planning as control of an ethnic minority. In: Sophie Watson / Katherine Gibson (Hg.): Postmodern cities and spaces. Cambridge (Massachusetts): Blackwell, 216-239.

Zaheer, Abasin (2014): Land grab: Wolesi Jirga summons Kabul mayor. In: Pajhwok Afghan News am 8.1.2014. https://www.pajhwok.com/en/2014/01/o8/wolesi-jirga-summonskabul-mayor (letzter Zugriff am 24.9.2018). 


\section{Informality from above and below: Urban development in Kabul framed by state-building and humanitarian interven- tion}

Urban Development in the Afghan capital after the Western intervention is framed by foreign-led state-building activities and its attendant appropriations of urban space by different actors. Against this background, the article aims to reconstruct spatial developments in Kabul after 2001 and to interpret these in the context of contemporary debates about urban informality in the Global South. In doing so, various interconnected regimes of urban planning that give rise to very specific productions of urban space are analysed using a framework of urban informality. The goal is to assess how urban inequality in Kabul is produced and maintained, how certain social dispositions and spatial practices can be explained in contexts of informality, and how urban informality itself can serve as resource for the accumulation of wealth and power. 